\title{
Disjoining-pressure-induced acceleration of mass shedding in solid-state dewetting
}

\author{
Ashwani K. Tripathi" and Olivier Pierre-Louis ${ }^{\dagger}$ \\ Institut Lumière Matière, UMR5306 Université Lyon 1-CNRS, Université de Lyon, 69622 Villeurbanne, France
}

(Received 26 July 2019; revised manuscript received 22 January 2020; accepted 27 February 2020; published 6 April 2020)

\begin{abstract}
Surface-diffusion mediated solid-state dewetting has been observed and studied in a number of different systems during the past two decades. This process can be accompanied by the pinching of the film at a finite distance from the retracting triple line. The repetition of this pinching is often referred to as periodic mass shedding. We show that the disjoining pressure of the film can accelerate mass shedding by orders of magnitude in ultrathin films with nanometric thickness. In the presence of power-law disjoining pressures induced by van der Waals forces, the mass shedding time exhibits an approximate power-law dependence on film thickness $t_{\mathrm{ms}} \sim \bar{h}^{\nu}$, with $v \approx 6$. Exponentially decaying disjoining forces also give rise to a strong acceleration of mass shedding. However, due to the finite range of the exponential potential, the mass shedding time does not exhibit a simple power-law dependence on the thickness, and is controlled by a cutoff thickness. In addition, two-dimensional simulations indicate that, within the range of thicknesses that we have studied and for isotropic dynamics, the transversal instability of a straight front does not lead to fingering, and mass shedding is the dominant instability of the dewetting front. Finally, we also show that no significant difference is observed in the dewetting dynamics between simulations based on a model with a wetting potential integrated over the film surface area, or over the projected substrate area.
\end{abstract}

DOI: 10.1103/PhysRevE.101.042802

\section{INTRODUCTION}

Thin solid films are one of the basic building blocks of many micro- and nanotechnologies. However, these films are often thermodynamically metastable or unstable. Indeed, when the temperature is high enough for surface diffusion mass transport to be activated, the morphology evolves spontaneously and islands are formed to minimize surface and interface energy. This process is called solid-state dewetting.

Experimental studies show that solid-state dewetting usually starts at defects or at the edges of the film $[1,2]$. Two model systems have been extensively studied in experiments: semiconductors on insulators $\left(\mathrm{Si}_{-} \mathrm{SiO}_{2}\right.$ or $\left.\mathrm{Ge}_{-} \mathrm{SiO}_{2}\right)$ [3-10] and metals on insulators $\left(\mathrm{Ni}-\mathrm{MgO}, \mathrm{Au}-\mathrm{SiO}_{2}\right.$, or $(\mathrm{Cu}$ or $\mathrm{Au}$ )-sapphire) [11-18]. During solid-state dewetting, complex morphological evolutions are observed where the film evolves via various kinetic mechanisms depending on the shape and orientation of defects. For example, depending on front orientation, the film either gives rise to pinching at finite distance from the dewetting front-a process which is usually called periodic mass shedding $[11,12,19]$, or through a transversal instability of the dewetting front giving rise to fingering $[3,6]$. Both processes (mass shedding and fingering) lead to the formation of linear structures which subsequently break up into solid islands via the Rayleigh-Plateau-like instability.

\footnotetext{
*Present address: Center for Soft and Living Matter, Institute of Basic Sciences, Ulsan 44919, Republic of Korea; ashwani.jnu@gmail.com

†olivier.pierre-louis@univ-lyon1.fr
}

The mass shedding mechanism does not occur in the dewetting of simple liquids where mass transport is mediated by hydrodynamics. Instead, mass transport in solid-state dewetting is usually mediated by surface diffusion, which produces an oscillatory tail behind the dewetting rim. The amplitude of the oscillations increases with time, ultimately leading to the pinching of the film. This process has been studied in detail by Wong et al. [19], and is similar to the process by which periodic hollow tori are produced during surface-diffusion mediated coalescence of spheres [20].

Our goal in this paper is to study the mass shedding mechanism in nanometrically thin films. Such ultrathin films exhibit a thickness-dependent free energy. The thickness-dependent part of the free energy is usually referred to as the wetting potential and its derivative as the disjoining pressure. The standard continuum models for solid-state dewetting describe the surface-diffusion-limited dynamics of thin solid films at thicknesses much larger than the range of the wetting potential. Hence diffusion on the film is not affected by the wetting potential, and the only effect of the wetting potential is summarized in the value of the contact angle $[19,21-$ 24]. Our approach is based on a continuum thin film model which is also valid at small thicknesses where the surface properties are directly influenced by the wetting potential. In order to do so, the standard Mullins model for surface diffusion is supplemented with a wetting potential which drives dewetting. We focus on the case of partial wetting, where the wetting potential exhibits a minimum, giving rise to a finite contact angle [25]. Thin film models with a contact angle as a boundary condition at the triple line and without influence of the wetting potential on the film away from the triple line are recovered from models with a wetting potential 
in the limit where the range of the wetting potential vanishes (see, e.g., Ref. [26]). Models with a wetting potential have already been used to study hole opening [27] in anisotropic solid films, and Ostwald ripening of nanoislands [28], or to derive effective nonequilibrium kinetic boundary conditions at the triple line of macroscopic films [26].

The standard interpretation of the wetting potential in the case of partial wetting is based on a combination of short-range repulsive forces resulting from the overlap of the regions of the film where bonds between atoms are affected by the surface and by the interface, and a long-range attractive force is attributed to van der Waals interactions. However, many different physical contributions can actually come into play [25], and we will therefore consider two generic situations, where the attractive part is either power law or exponential.

We show that the mass shedding process in solid-state dewetting, which exists even when the range of the wetting potential vanishes, is accelerated by orders of magnitude in the presence of a wetting potential, even if the range of the wetting potential is much smaller than the initial film thickness. This is different from the case of liquid dewetting, where no mass shedding is found when the range of the wetting potential vanishes, but an instability similar to mass shedding is found when the range of the wetting potential is of the same order as the film thickness, as discussed for example in Refs. [29,30].

The mass shedding time in solid-state dewetting exhibits a power-law dependence on the film thickness in the case of attractive van der Waals forces. In contrast, no power law is observed for exponentially decaying potentials. We propose that the pinching time is controlled by a cutoff thickness in the case of exponential potentials. For film thicknesses of the order of the range of the wetting potential, the film destabilizes everywhere away from the film edges due to the spinodal instability. We also briefly report on the full two-dimensional dynamics of the mass shedding process. These simulations reveal that no fingering instability takes place for very thin films with isotropic dynamics.

The paper is organized as follows. In Sec. II, we present our one-dimensional mesoscopic model. Section III reports on the acceleration of periodic mass shedding for the van der Waals potential. In Sec. IV, we summarize the results for the exponential potential. Section V presents some numerical simulations for two-dimensional dynamics. Finally, we discuss our results in the light of existing experiments and conclude in Sec. VI.

\section{WETTING POTENTIAL MODEL}

\section{A. Free energy}

In this paper, we discuss the diffusion-limited dynamics of thin solid films. We neglect elastic effects, and the solid is considered to be rigid.

Let us start by defining $\gamma_{\text {int }}$ - the free energy per unit area of the interface between two different semi-infinite solids. Then, consider a flat solid film of thickness $h$ made of one of these solids on a semi-infinite bulk made of the other solid. Discarding bulk contributions (proportional to the volume) the free energy of the film per unit area is a function of the film thickness denoted as $\phi(h)$. We define $\gamma(h)=\phi(h)-\gamma_{\text {int }}$, and decompose it into a constant term and an $h$-dependent term

$$
\gamma(h)=\bar{\gamma}+w(h)
$$

where $w(h) \rightarrow 0$ as $h \rightarrow+\infty$, so that $\phi(h)=\gamma_{\text {int }}+\bar{\gamma}$ as $h \rightarrow+\infty$. As a consequence, $\bar{\gamma}$ can be interpreted as the asymptotic surface energy of the film for large $h$. The wetting potential $w(h)$ arises due to many physical effects such as breaking of chemical bond extending farther than nearest neighbors, structural effects, electrostatic effects, and van der Waals interactions [25,31].

Based on the free energy $\gamma(h)$, we now wish to build a model energy for a one-dimensional film with a thickness $h(x, t)$ depending on the space coordinate $x$ and the time $t$. Here, we assume that the film thickness does not depend on the spatial coordinate $y$ orthogonal to $x$. Keeping an isotropic surface free energy $\gamma$ which depends on $h$ but not on $\partial_{x} h$ and higher order derivatives, the total free energy per unit length along $y$ reads

$$
\mathcal{F}=\int d s \gamma(h),=\int d s[\bar{\gamma}+w(h)],
$$

where $s$ is the arclength coordinate along the surface profile $h(x, t)$ and

$$
d s=\left[1+\left(\partial_{x} h\right)^{2}\right]^{1 / 2} d x .
$$

We remark that the free energy Eq. (2) is not the only possible model which extends the definition of the wetting potential to finite slopes. Indeed, while some authors have used an approach similar to ours to study wetting statics and dynamics at finite slopes [26,27,32-36], other options are possible as reported, e.g., in Ref. [37], where the finite-slope wetting term is chosen as $\int d x w(h)$ instead of $\int d s w(h)$. On the one hand, the integration over $d x$ leads to a contribution in the chemical potential which is simple and identically equal to that of the small slope limit. On the other hand, as discussed in Ref. [38], integration over $d s$ is very convenient for example to add controlled anisotropy in solid-state wetting (due to the isotropy of the reference model). However, none of these models are derived from a rigorous asymptotic expansion. Expressions of the free energy based on $h$ and its spatial derivatives have only been obtained as an expansion, as discussed, e.g., in small slope expansions from Ref. [39] for van der Waals-like interactions, or in Ref. [40] for surface energy anisotropy. Furthermore, we show in Appendix I that the two energy models (with $d x$ or $d s$ ) do not lead to quantitatively significant differences for our dewetting simulations. This quantitative similarity has been already noticed in the context of liquidstate wetting in the static regime in Ref. [36]. In the following, we use the model energy Eq. (2).

We consider a wetting potential $w(h)$ with a well defined stable state at $h=h_{m}$ corresponding to a wetting film or to a bare substrate with an adsorbate layer, as discussed, e.g., in Ref. [28]. We focus on the case of partial wetting, where the wetting potential, shown in Fig. 1, exhibits a single minimum at $h=h_{m}$ with $w\left(h_{m}\right)<0$. A balance of tensions at equilibrium then leads to the Young-Dupré equation for the equilibrium contact angle $\theta_{\mathrm{eq}}$, where $\theta=\arctan \left(\partial_{x} h\right)$. This relation reads $\bar{\gamma} \cos \theta_{\mathrm{eq}}=\gamma\left(h_{m}\right)$, and may be rewritten with 

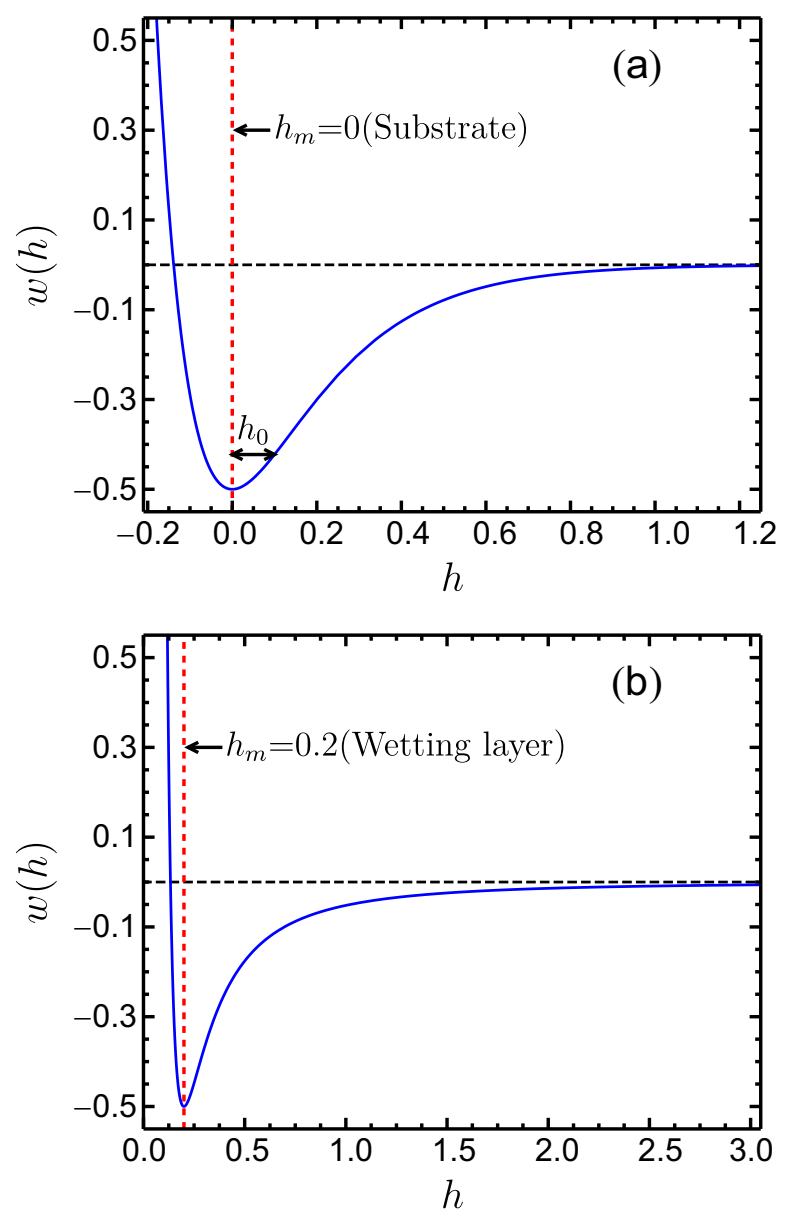

FIG. 1. Wetting potential as function of film thickness. (a) Exponential case with a minimum at $h_{m}=0$ and a width $h_{0}$. (b) van der Waals potential with a minimum at $h_{m}>0$.

the wetting potential as

$$
-w\left(h_{m}\right)=\bar{\gamma}\left(1-\cos \theta_{\mathrm{eq}}\right) .
$$

Hence the partial wetting regime for which $\theta_{\mathrm{eq}}>0$ corresponds to $w\left(h_{m}\right)<0$. In addition, we do not allow for overhangs. Therefore, all angles, including the equilibrium contact angle $\theta_{\mathrm{eq}}$, must be smaller than $90^{\circ}$. Inserting this condition in Eq. (4), we have $w\left(h_{m}\right)>-\bar{\gamma}$ and the total free energy $\gamma(h)=\bar{\gamma}+w(h)$ is always positive.

The local chemical potential is derived from the cancellation of the variation $\delta(\mathcal{F}-\mu \mathcal{N})=0$. We have defined the number of atoms per unit length along $y \mathcal{N}=\int d x h / \Omega$ and the volume per atom $\Omega$. We therefore obtain $\mu=$ $(\delta \mathcal{F} / \delta h) /(\delta \mathcal{N} / \delta h)$, where $(\delta \bullet / \delta h)$ denotes the functional derivative. This leads to

$$
\mu=\Omega\left(\gamma(h) \kappa+\frac{\gamma^{\prime}(h)}{\left[1+\left(\partial_{x} h\right)^{2}\right]^{1 / 2}}\right),
$$

where $\gamma^{\prime}(h)$ denotes the derivative of $\gamma(h)$ with respect to $h$, and the curvature of the surface reads

$$
\kappa=\frac{-\partial_{x x} h}{\left[1+\left(\partial_{x} h\right)^{2}\right]^{3 / 2}} .
$$

In equilibrium, the chemical potential $\mu$ must be independent of time and space. Using this condition, multiplying Eq. (5) by $\partial_{x} h$, and integrating, one obtains the Young-Dupré equation (4), as discussed for example in Refs. [25,26].

\section{B. Surface-diffusion dynamical model}

We now turn to the dynamical model for surface-diffusionlimited dynamics. The nonequilibrium states are characterized by a spatially nonhomogeneous local chemical potential. In a long-wave approach, the nonequilibrium thermodynamic driving force is then proportional to the gradient of chemical potential $\partial_{s} \mu$. Within the framework of linear irreversible processes, we assume that the surface mass flux $j$ is proportional to the driving force and we write $j=-m \partial_{s} \mu$, where the kinetic coefficient $m$ is a surface mobility. Finally, due to local mass conservation, the film surface evolves via conserved dynamics, and the normal velocity $v_{n}$ of the surface is written as the divergence of the surface mass flux $v_{n}=-\partial_{s} j$. Using the expression of $v_{n}$,

$$
v_{n}=\frac{\partial_{t} h}{\left[1+\left(\partial_{x} h\right)^{2}\right]^{1 / 2}},
$$

and Eq. (3) the evolution equation of the film thickness in Cartesian coordinates reads

$$
\partial_{t} h=\partial_{x}\left[\frac{\Omega m}{\left[1+\left(\partial_{x} h\right)^{2}\right]^{1 / 2}} \partial_{x}\left(\gamma(h) \kappa+\frac{\gamma^{\prime}(h)}{\left[1+\left(\partial_{x} h\right)^{2}\right]^{1 / 2}}\right)\right] .
$$

In the following, we assume for the sake of simplicity that $m$ is a constant independent of $h$. Some discussion of the case where $m$ depends on $h$ can be found in Ref. [26].

All one-dimensional simulations in this paper are based on the numerical solution of the finite-slope model Eq. (8) with different expressions of the wetting potential. For the numerical integration, we use the implicit Euler pseudospectral numerical scheme discussed in Ref. [26].

\section{Small slope limit}

The local surface angle is defined as $\theta=\arctan \left(\partial_{x} h\right)$. In the small slope limit, we have $\partial_{x} h \sim \theta \ll 1$. Thus Eq. (4) indicates that the wetting potential is small as compared to the asymptotic surface energy $w \sim \bar{\gamma} \theta^{2} \ll \bar{\gamma}$. As a consequence, the evolution equation of the film assumes a simple expression to leading order [26]

$$
\partial_{t} h=m \Omega\left[-\bar{\gamma} \partial_{x}^{4} h+\partial_{x x} w^{\prime}(h)\right] .
$$

Our analytical discussions of the simulation results are mainly based on the small slope limit Eq. (9). Indeed, the small slope equations are simpler and are therefore easier to analyze analytically. Furthermore, the small slope limit often leads to predictions that are quantitatively not too far from the finite slope models (see, e.g., Ref. [19]). Finally, the small slope limit is in some sense more universal than finite slope models (for example, both finite slope models discussed above in Sec. II A, corresponding to integration of $w$ along $d x$ or $d s$, exhibit the same small slope limit).

However, as mentioned in the previous subsection, we use the finite-slope model Eq. (8) and finite contact angles in all simulations. Indeed, most experimental systems exhibit finite contact angles, i.e., finite slopes. We therefore aim to describe the behavior at finite slopes to be able to discuss experimental 
results. In addition, numerical simulations with finite contact angles - and therefore with finite slopes - are usually faster than those with small contact angles. We therefore use finiteslope simulations to simulate larger systems and to have an easier access to long-time regimes.

\section{VAN DER WAALS WETTING POTENTIAL}

\section{A. Model and simulations}

We first consider a potential with a power-law attractive van der Waals contribution $h^{-2}$ [31]

$$
w(h)=\bar{\gamma}\left(1-\cos \theta_{\mathrm{eq}}\right)\left[2\left(\frac{h_{m}}{h}\right)^{3}-3\left(\frac{h_{m}}{h}\right)^{2}\right] .
$$

The power-law form of the repulsion term $h^{-3}$ is chosen for convenience, and is similar to that used for liquid dewetting studies. The precise dependence of the repulsive term on $h$ is not relevant for our conclusions, as long as it obeys two conditions: (i) it is negligible as compared to the attractive part for $h \gg h_{m}$ and (ii) it is large enough at small $h$ to lead to a minimum at $h=h_{m}$. The attractive part of the wetting potential is chosen to scale as $h^{-2}$. This dependence is the most used to describe van der Waals forces; however, other behaviors are possible (see, e.g., Refs. [25,31]).

The wetting potential produces an equilibrium contact angle obeying the Young-Dupré relation (4). Since the wetting potential diverges for $h \rightarrow 0$, negative thicknesses are forbidden. Note that the length scale $h_{m}$ describes both the minimum of $w$ and the width of the potential well. Within this model, $h_{m}$ can be interpreted as the width of a wetting or adsorbate layer.

We have investigated the evolution of a thin film via the numerical integration of Eq. (8). We have used $h_{m}=0.2$ and equilibrium contact angle $\theta_{\mathrm{eq}}=60^{\circ}$ in our simulations. The spatial discretization length was $d x=\pi / 32$ and the time step was $d t=0.002$. The initial condition is a (exponential) step function with a film thickness $\bar{h}$ ranging from $5 h_{m}$ to $30 h_{m}$. The film thickness $\bar{h}$ is defined as the difference between the height of the flat film and the height of the wetting layer $h_{m}$.

The films exhibit the expected behavior as reported in Fig. 2(b). First, a rim forms at the edge of the solid film where the mass accumulates. The rim exhibits an oscillatory tail in the film region. The first minimum of these oscillations deepens with time, and ultimately leads to a pinching event. This process then repeats in time, giving rise to the periodic mass shedding scenario [19]. In the following, we focus on the behavior of the first minimum of the oscillations of the profile away from the triple line, which is involved in the pinching event.

\section{B. Two regimes}

Let us define the position $x_{\min }(t)$ of the minimum of the first oscillation of the film profile after the triple line. In the following, we use the index min to indicate quantities evaluated at $x=x_{\min }(t)$. As an example, $h_{\min }=h\left(x_{\min }(t), t\right)$. Since $\partial_{x} h_{\min }=0$, the small slope limit Eq. (9) leads to

$$
\partial_{t} h_{\min }=m \Omega\left[-\bar{\gamma} \partial_{x}^{4} h_{\min }+w^{\prime \prime}\left(h_{\min }\right) \partial_{x x} h_{\min }\right] .
$$
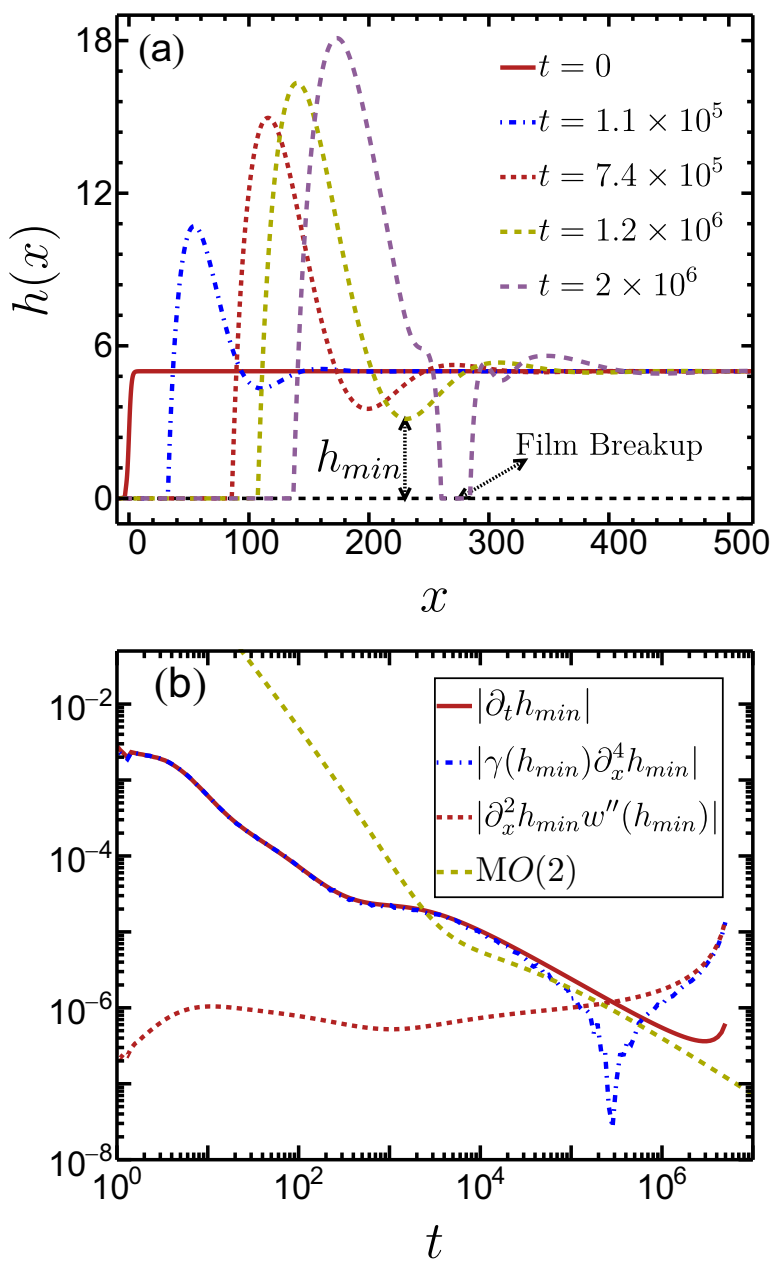

FIG. 2. Dewetting dynamics at the edge of a solid film. (a) Evolution of a film profile starting from a step. (b) Time dependence of $\partial_{t} h_{\min }$, for $\bar{h}=6.0$. "Mullins term" and the "Wetting term" denote the contributions to $\partial_{t} h_{\text {min }}$ originating from surface curvature and wetting potential, respectively [see Eq. (11)]. $\mathrm{M} O(2)$ is the second order solution for $\partial_{t} h_{\min }$, obtained from the small slope approximation of the Mullins model in the long-time limit [19].

The comparison of the two terms in the right hand side (RHS) of this equation with $\partial_{t} h_{\mathrm{min}}$ in the simulations suggests two regimes. Initially, the film thickness in the region of the first minimum is too large to be affected by the wetting potential and the film evolution is driven by surface tension. This regime is observed approximately from $t=10$ to about $10^{5}$ in Fig. 2(b), when the (brown) solid line corresponding to the value of $\left|\partial_{t} h_{\min }\right|$ observed in simulations collapses with the (blue) dotted-dashed line corresponding to the value of the Mullins term $\bar{\gamma} \partial_{x}^{4} h_{\min }$ calculated at the minimum. We refer to this regime as the Mullins regime. Then, we observe a second regime where the wetting potential and the Mullins term are of the same order. This regime ends with the detachment of the first oscillation of the rim from the rest of the film.

In Appendix H, we derive the finite-slope generalization of Eq. (11), which contains three additional terms. A numerical evaluation, presented in Fig. 17, shows that these three terms are negligible as compared to the two terms in the RHS of Eq. (11). We conclude that Eq. (11) provides a satisfactory 
approximation of the dynamics at the minimum during dewetting processes governed by Eq. (8). However, finite slope effects are expected to lead to deviations from the small slope limit in other parts of the profile. For example, close to the triple line, the slope approaches the finite equilibrium contact angle. Hence the dynamics is still globally influenced by finite-slope effects.

\section{Mullins regime}

Let us now analyze the Mullins regime in more detail. When the potential term is negligible, Eq. (11) suggests a scaling relation $x^{4} \sim t$ which is usual in surface-diffusion limited dynamics. In addition, the condition of a fixed contact angle $\theta_{\text {eq }}$ and a fixed scale for the heights selected by the initial thickness $\bar{h}$ imposes the relation $x \sim \bar{h} / \theta_{\text {eq }}$. This suggests a scaling variable $t / x^{4} \sim t \theta_{\mathrm{eq}}^{4} / \bar{h}^{4}$. Discarding the dependence in $\theta_{\text {eq }}$, we obtain a scaling ansatz of the form

$$
\bar{h}-h_{\min }(t)=\bar{h} \mathcal{F}\left(t / \bar{h}^{4}\right) .
$$

As shown in Fig. 3, data collapse of simulation results based on Eq. (12) is achieved in an intermediate regime between a first transient relaxation and the final stages of pinching when the thickness around the minimum is small enough for the wetting potential to become relevant.

Within this scaling regime, the analytical asymptotic expansion presented in Ref. [19] provides the surface profile during dewetting in the limit of small slopes and large times (see also Ref. [41]). The scaling function $\mathcal{F}$ can be extracted from this result, and takes the form

$$
\mathcal{F}(\xi) \approx a \xi^{1 / 5}+b+c / \xi^{1 / 5}+\cdots
$$

where $\xi=t / \bar{h}^{4}$ for large times. The details of the analytical expression are reported in Appendix A. The scaling behavior Eq. (12) and the scaling function Eq. (13) were shown to be quantitatively accurate in the limit of small slopes in Ref. [19] using numerical simulations of the Mullins model without wetting potential [i.e., Eq. (8) with $\gamma(h)=\bar{\gamma}$, and $w(h)=0$ ], and with a fixed contact angle $\theta_{\mathrm{eq}}$ imposed at the triple line.

As shown in Fig. 2(b), the prediction Eq. (13) approaches asymptotically our results at long times, but does not agree with them quantitatively. However, as seen in Fig. 3, the convergence to the scaling function $\mathcal{F}(\xi)$ during the Mullins regime improves when the film thickness $\bar{h}$ is increased. The values of $\xi$ reached in our simulations are not large enough for the behavior of $\mathcal{F}(\xi)$ to be dominated by the first term in Eq. (13). This could happen only for $\xi$ larger than $10^{3}$, which is beyond the range of numerical simulations. Similarly, a simple power-law fit of the scaling function $\mathcal{F}(\xi)=a+b \xi^{c}$ leads to $c \approx 0.36$, a value which is significantly larger than the expected asymptotic exponent $1 / 5$.

As a summary, our results show a Mullins regime with a scaling behavior in agreement with Eq. (12). The simulation results approach the scaling function Eq. (13). Nonetheless, the separation of scales $\bar{h} \gg h_{m}$ is not large enough in our simulations to reach an asymptotic behavior described only by the asymptotic exponent $1 / 5$.

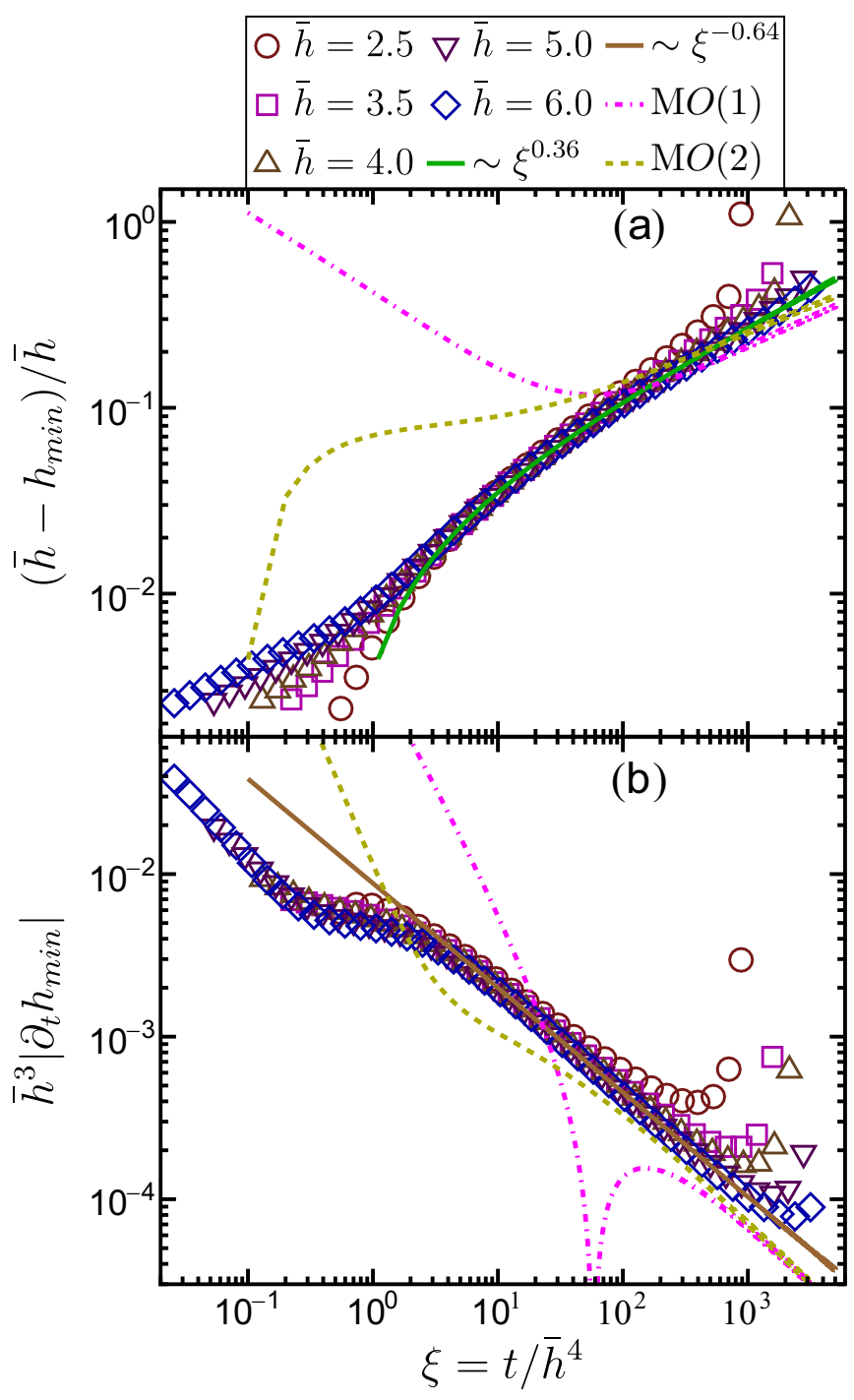

FIG. 3. Data collapse for $h_{\min }$ and $\partial_{t} h_{\min }$ in the Mullins regime. Data are scaled according to Eq. (12). Solid lines are fit to the data according to the function $\mathcal{F}(\xi)=a+b \xi^{c}$. $\mathrm{M} O(1)$ and $\mathrm{M} O(2)$ represent respectively the first and second order solutions of the small slope approximation of Mullins' model in the long time limit with $\alpha_{0}=\theta_{\mathrm{eq}}$.

\section{Potential-dominated regime}

The Mullins regime ends when the wetting potential starts to affect the dynamics of the first minimum of the profile. As a first insight on the influence of the wetting potential, we study the stability of linear perturbations of a flat film far from the edges. Consider a small perturbation of the film profile around a flat film $\delta h(x, t)=h(x, t)-\bar{h} \ll \bar{h}$. Substituting this expression into Eq. (8) or Eq. (9), and linearizing, we obtain a linear equation with constant coefficients. This equation provides a dispersion relation for Fourier modes $\delta h_{q \omega} \sim e^{i \omega t+i q x}$. In the small slope limit Eq. (9), we obtain

$$
i \omega=m \Omega\left[-\bar{\gamma} q^{4}-w^{\prime \prime}(\bar{h}) q^{2}\right] .
$$

In the more general case where finite slopes are allowed, Eq. (8) leads to

$$
i \omega=m \Omega\left[-\gamma(\bar{h}) q^{4}-w^{\prime \prime}(\bar{h}) q^{2}\right] .
$$


The Fourier modes will grow when $\operatorname{Re}(i \omega)>0$, i.e., when $w^{\prime \prime}(\bar{h})<0$. This is the well-known spinodal instability which appears at long wavelengths for $q<\left[-w^{\prime \prime}(\bar{h}) / \gamma(\bar{h})\right]^{1 / 2}$. The timescale of the appearance of the instability is defined as $t^{\mathrm{SD}}=2 \pi / \max _{q}[\operatorname{Re}(i \omega)]$ and reads

$$
t^{\mathrm{SD}}=\frac{8 \pi \gamma(\bar{h})}{m \Omega\left|w^{\prime \prime}(\bar{h})\right|^{2}}
$$

Since we consider a potential which is attractive at long distances, we have $w^{\prime \prime}(\bar{h})<0$ and this instability is expected to be present.

However, pinching is a spatially localized event and the linear stability of a flat film is not appropriate to study this effect. Instead, we have searched for a localized self-similar solution in the pinchoff regime under the influence of the wetting potential, following the same line as some previous studies on liquid dewetting [42-44]. For this purpose, we consider a self similar ansatz

$$
h(x, t) \sim\left(t_{\mathrm{ms}}-t\right)^{1 / \beta} f\left(\frac{x-x_{\mathrm{ms}}}{\left(t_{\mathrm{ms}}-t\right)^{\alpha}}\right) .
$$

Substituting this solution in Eq. (9) and considering that the wetting potential is dominated by the attractive part of the van der Waals potential for $h \gg h_{m}$, we find a self-similar solution for $\alpha=1 / 4$ and $\beta=8$ (the derivation of these exponents is reported in Appendix B, where it appears as a special case of a discussion for arbitrary power laws $w \sim-h^{-n}$ ). When $h=h_{\min }$ Eq. (17) reduces to

$$
h_{\min }(t)=\left(\frac{A \Omega^{1 / 2} m^{1 / 2}}{\bar{\gamma}^{1 / 2}}\right)^{1 / 4}\left(t_{\mathrm{ms}}-t\right)^{1 / 8} f(0),
$$

where $f(0)$ is a dimensionless constant and $A=3 \bar{\gamma} h_{m}^{2}(1-$ $\left.\cos \theta_{\text {eq }}\right)$.

We have rescaled the numerical data for $h_{\min }$ with the selfsimilar ansatz of Eq. (17). This is shown in Fig. 4. The best data collapse is obtained for $\beta \approx 7.2$, which deviates slightly from the expected value $\beta=8$. This deviation could result from the difficulty to reach the separation of scales $h \gg h_{m}$ in the simulations leading to a contribution of the repulsive part of the potential which is neglected in the analysis of the scaling behavior, and which increases rapidly when the film surface is very close to the substrate. Fitting the numerical data with $\beta=8$ provides $f(0)=1.07$ (see Fig. 12 in the Appendixes).

\section{E. Total pinching time}

The total mass shedding time cumulates both processes of formation of the minimum with Mullins dynamics and of final pinching controlled by the wetting potential. As a simple approximation, we therefore propose to evaluate the total mass shedding time $t_{\mathrm{ms}}$ as the sum of the time $t_{*}$ that the system spends in the Mullins regime and the time $t_{\mathrm{ms}}-t_{*}$ of the final pinching regime. This approach requires one to match the two solutions at the time $t_{*}$. In order to do so, we impose both that the thickness at the minimum and its time derivative are equal in the two solutions at $t_{*}$. The resulting expressions are rather tedious, and are reported in Appendix C. As shown in Fig. 5, the best quantitative agreement of this approach with the numerical data of $t_{\mathrm{ms}}$ is obtained for $f(0) \approx 1.4$ when

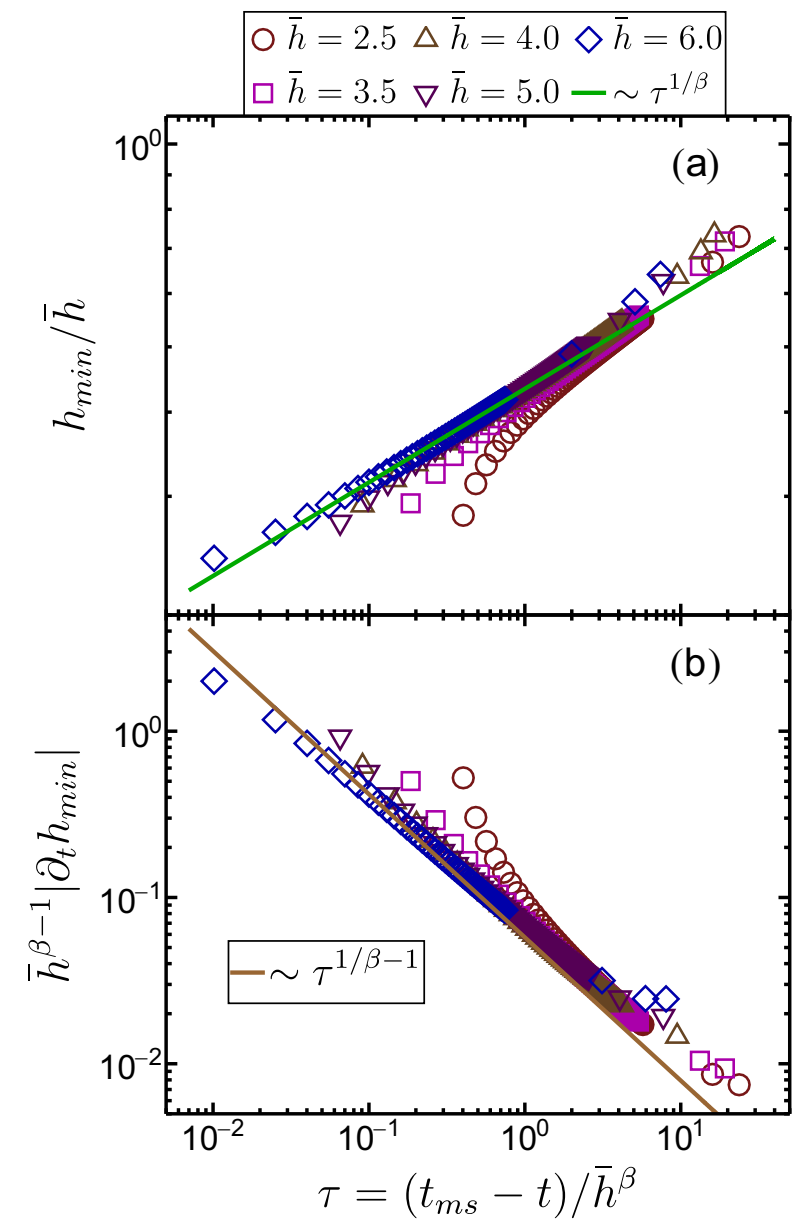

FIG. 4. Data collapse for $h_{\min }$ and its derivative in the pinchoff regime. Data are scaled according to the self-similar solution described in the text with $\beta=7.2$. The continuous lines correspond to the fit to collapsed data using Eq. (17) with $\beta$ and $f(0)$ as fitting parameter.

using $\alpha_{0}=\theta_{\text {eq }}$. As shown in Appendix F, using $\alpha_{0}=\tan \theta_{\text {eq }}$ leads to $f(0)=1.05$. We except the discrepancies between these two values of $f(0)$, and the value $f(0)=1.07$ obtained above from the fitting of the data collapse of $h_{\min }$, to be smaller in the small slope limit. They may differ here because the simulations are performed with a finite contact angle $\theta_{\text {eq }}=60^{\circ}$.

A direct power-law fitting of the total time for mass shed$\operatorname{ding} t_{\mathrm{ms}}$,

$$
t_{\mathrm{ms}}=\bar{h}^{\nu}
$$

with $v \approx 6$, shown in Fig. 5, is in good agreement with the numerical simulations. This exponent is intermediate between two extreme cases. First, if the dynamics was within the Mullins regime up to pinching, then we could use Eq. (12) with $h_{\min }=0$, leading to $t_{\mathrm{ms}} \sim \bar{h}^{4}$ [19]. In contrast, if the dynamics was dominated by the pinch-off regime we could substitute $h_{\min }$ by $\bar{h}$ and $t$ by zero in Eq. (18), and we would obtain $t_{\mathrm{ms}} \sim \bar{h}^{8}$. The observed exponent $v \approx 6$ results from a compromise between these two limits.

The corresponding nonaccelerated mass-shedding time obtained in absence of wetting potential [19] is reported in the 


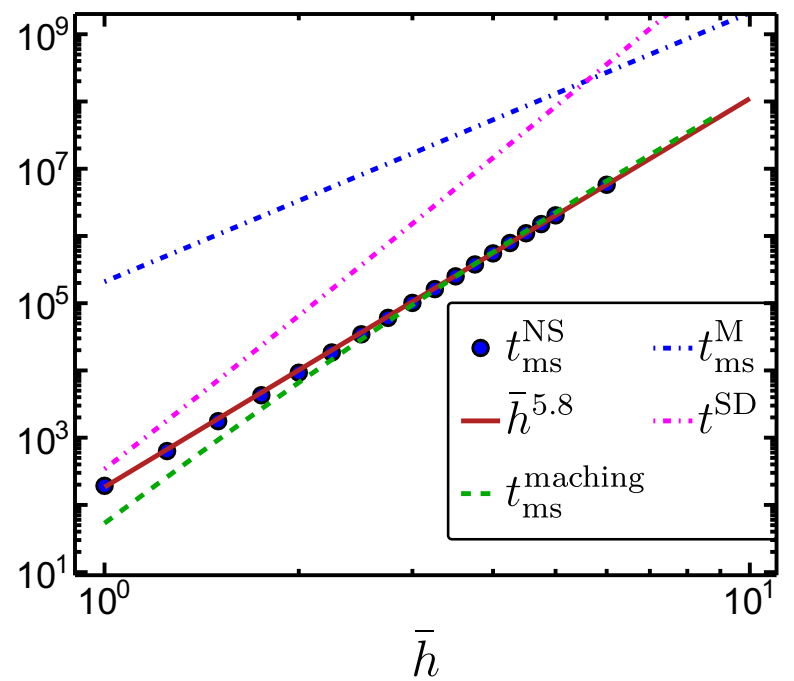

FIG. 5. Mass shedding time from simulations $t_{\mathrm{ms}}^{\mathrm{NS}}$ as a function of film thickness for van der Waals potential. Here, $t_{\mathrm{ms}}^{\mathrm{M}}$ is the mass shedding time obtained in the absence of wetting potential and $t^{\mathrm{SD}}$ is the timescale of spinodal dewetting. $t_{\mathrm{ms}}^{\text {matching }}$ denotes the mass shedding time obtained from matching the solution without wetting potential with the pinch-off solution (performed with $\alpha_{0}=\theta_{\text {eq }}$ ). The continuous line denotes the power law fit to the numerical data.

same figure. The numerical results indicate that the wetting potential can accelerate the mass shedding process by at least $10^{3}$ or $10^{4}$ times. This acceleration is larger at small thicknesses.

We have also reported the timescale of spinodal dewetting for which the film is unstable to random fluctuations at the film surface on Fig. 5. The timescale of spinodal dewetting is comparable to that of mass shedding at small thicknesses. Hence we expect a competition between spinodal dewetting and mass shedding at very small thicknesses. However, the mass shedding scenario dominates at larger thicknesses.

\section{EXPONENTIAL WETTING POTENTIAL}

Let us now consider the case where short-range interactions dominate. In order to study this case, we consider a potential which decays exponentially for large thicknesses

$$
w(h)=\bar{\gamma}\left[1-\cos \left(\theta_{\mathrm{eq}}\right)\right]\left[e^{-\left(h-h_{m}\right) / h_{0}}-2 e^{-\left(h-h_{m}\right) /\left(2 h_{0}\right)}\right] .
$$

This potential exhibits a minimum at $h=h_{m}$, and extends up to a range of the order of $h_{0}$. In the following we will consider $h_{m}=0$ without loss of generality (up to a shift in the definition of $h$ ). The numerical integration parameters were $d x=\pi / 16$ and $d t=0.01$.

In simulations with $h_{0}=0.1$, we observe once again two different regimes in the mass shedding process: a Mullins regime where the influence of the wetting potential is negligible and a second regime when the wetting potential plays a major role.

The time evolution of $h_{\min }$ and its time derivative are summarized in Fig. 6. A good data collapse in Figs. 6(b) and 6(c) confirms the scaling ansatz Eq. (12) and indicates that the evolution is in the Mullins regime at intermediate times (except for the very thin film with $\bar{h}=1$, where the regimes
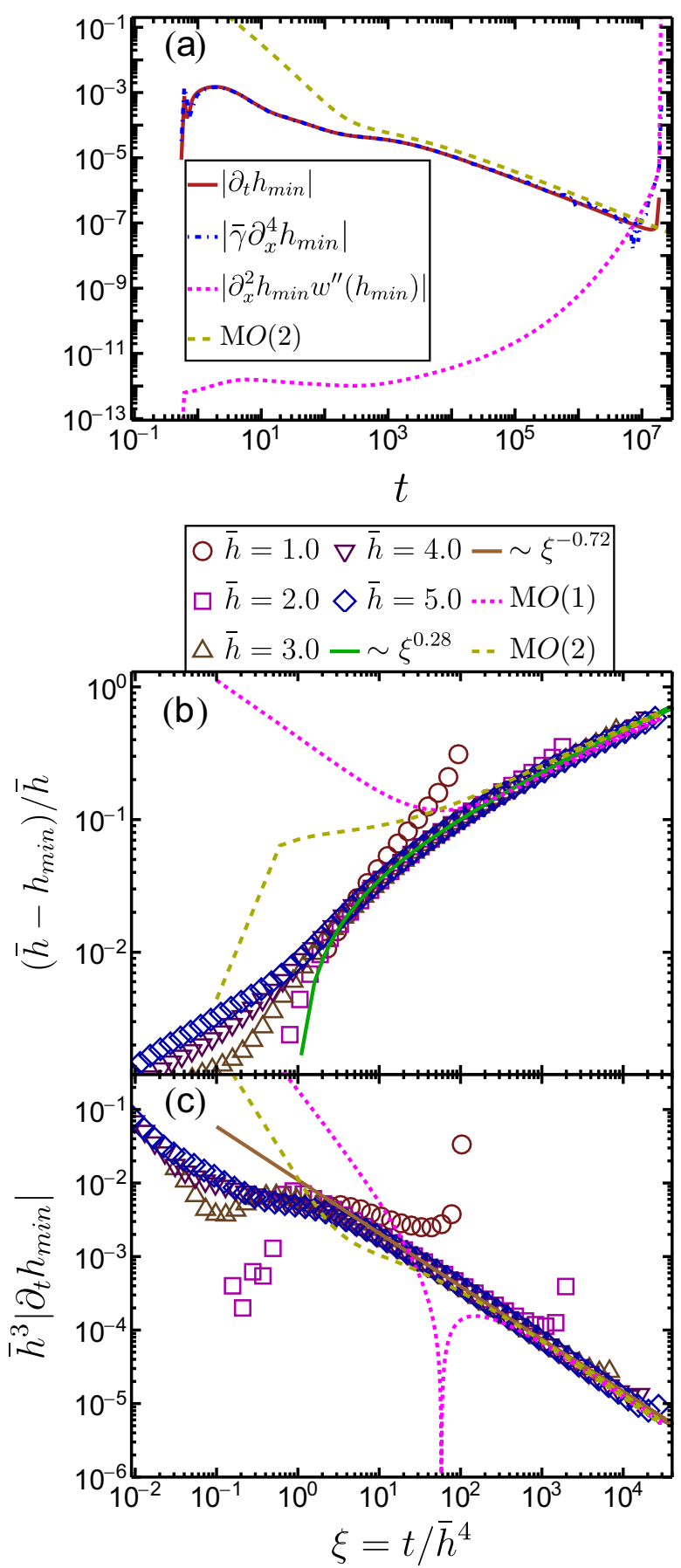

FIG. 6. Evolution of the minimum of the profile for the exponential wetting potential. (a) Time evolution of $\partial_{t} h_{\min }$ for $\bar{h}=$ 5.0 , together with the contributions of the "Mullins term" and the "Wetting term." (b),(c) Data collapse of $h_{\min }$ and $\partial_{t} h_{\min }$ in the Mullins regime. Data are scaled according to Eq. (12). The green and brown curves represent a fit to the collapsed data using $\mathcal{F}(\xi)=a+b \xi^{c}$.

are not well separated in time). Furthermore, comparison of Fig. 6(a) with Fig. 2 suggests that the evolution with an exponential potential exhibits a faster convergence towards the scaling function Eq. (13) corresponding to the solution of Wong et al. [19]. In addition, as shown in Figs. 6(b) and 6(c), a fit of the scaling function of the form $\mathcal{F}(\xi)=a+b \xi^{c}$ provides an exponent $c=0.28$, which is closer to asymptotic 


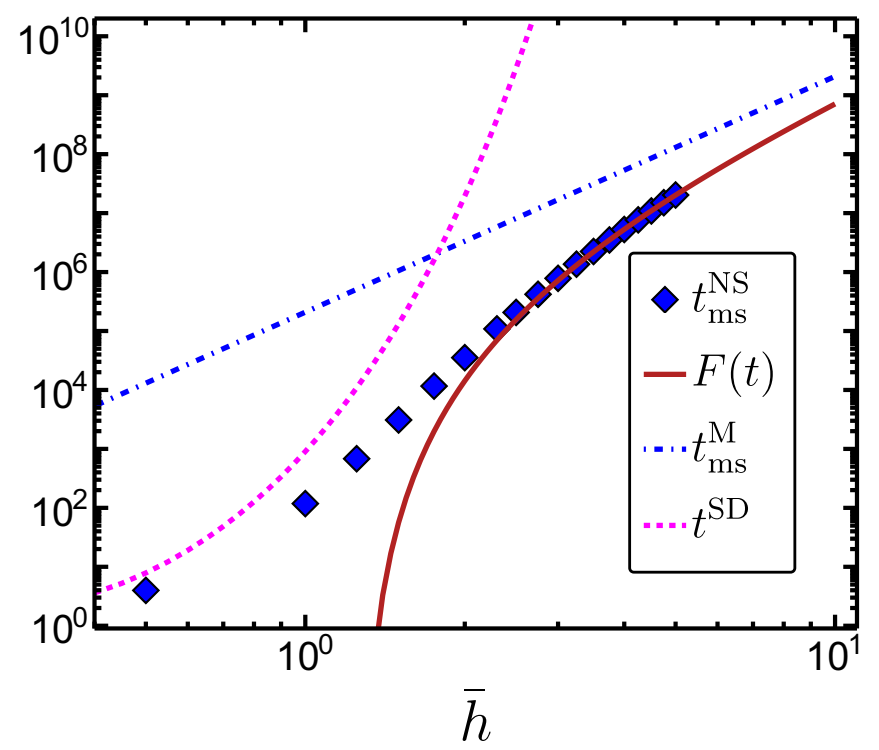

FIG. 7. Mass shedding time $t_{\mathrm{ms}}^{\mathrm{NS}}$ as a function of film thickness for exponential potential. Here, $t_{\mathrm{ms}}^{\mathrm{M}}$ is the mass shedding time in the absence of wetting potential and $t^{\mathrm{SD}}$ is the timescale of spinodal dewetting. The continuous line denotes the fit to the numerical data according to Eq. (21) with $t_{\exp }=0$ and $h_{c}=1.5$.

prediction $1 / 5$ than in the case of the van der Waals potential. This faster convergence could be caused by a faster decrease of the exponential potential when increasing film thickness.

We have also examined the variation of total mass shedding time $t_{\mathrm{ms}}$ with film thickness, as reported in Fig. 7. We observe that $t_{\mathrm{ms}}$ is at least $10^{2}$ to $10^{4}$ times smaller than the corresponding value for the Mullins model without any wetting potential. When the film thickness is decreased the acceleration of the shedding process increases very rapidly, i.e., faster than a power law. Indeed, due to the exponential form of the potential, we do not expect a self-similar pinching solution in the form of Eq. (17).

A simple argument allows one to gain further insight on this behavior. Indeed, we assume that the exponential wetting potential only influences the dynamics at a finite distance $h_{c}$ from the substrate which is controlled by the range $h_{0}$ of the potential. Once the thickness has reached $h_{c}$, the pinching takes a finite time $t_{\mathrm{exp}}$. This simple picture neglects the details of the matching between the Mullins regime and the final pinching regime. However, since the potential is exponentially decreasing with $h$, the details of the matching should only bring logarithmic corrections. Here, we neglect these corrections and, using the expression of $h_{\min }$ in Eq. (12) [see also the full expression Eq. (A2)], we find

$$
t_{\mathrm{ms}}=t_{\mathrm{exp}}+\frac{\bar{h}^{4}}{m \Omega \bar{\gamma} \alpha_{0}^{4}}\left(C_{1}-C_{2} \frac{h_{c}}{\bar{h}}\right)^{5},
$$

where $C_{1}=12.0204 \ldots$ and $C_{2}=9.3478 \ldots$ are numerical constants, the expressions of which are reported in Appendix (D2). Assuming $t_{\exp } \approx 0$ and $\alpha_{0}=\theta_{\text {eq }}$, we use Eq. (21) to fit the numerical results with two fitting parameters $C_{1}$ and $C_{2} h_{c}$. This provides a value $C_{1}=11.11$, and using the value of $C_{2}$ from analytical expression we obtain the cutoff height $h_{c} \approx$ 1.5. The results are shown in Fig. 7. If we use $\alpha_{0}=\tan \theta_{\mathrm{eq}}$ instead of $\alpha_{0}=\theta_{\mathrm{eq}}$, fitting the data for $t_{\mathrm{ms}}$ using Eq. (21) provides $C_{1}=16.62$ and $h_{c}=2.27$. These small quantitative discrepancies again originate in an application of the small slope limit beyond its regime of validity.

Finally, as compared to the van der Waals potential, the ratio of the spinodal instability time over the mass shedding time increases much faster in the exponential case, as seen in Fig. 7. This is clearly a consequence of the fast decay of the exponential potential for large $h$.

\section{2D SIMULATIONS}

In the previous sections, we have investigated the dewetting dynamics of a thin solid film within a one-dimensional model. In this section, we briefly present numerical results for the full two-dimensional dynamics of the dewetting process. As a straightforward generalization of the model Eq. (8), we consider a finite-slope two-dimensional surface with a height profile $h(x, y)$, which obeys

$$
\partial_{t} h=\nabla \cdot\left[\frac{\Omega m}{\left(1+|\nabla h|^{2}\right)^{1 / 2}} \nabla\left(\gamma(h) \kappa+\frac{\gamma^{\prime}(h)}{\left(1+|\nabla h|^{2}\right)^{1 / 2}}\right)\right],
$$

where $\nabla$ denotes the gradient operator in the $(x, y)$ plane. Again, we consider the case of a constant mobility $m$. The numerical integration scheme is similar to that of Ref. [26], with an implicit Euler pseudospectral method. All simulations of the finite-slope model Eq. (22) are performed on a periodic discrete $1024 \times 1024$ grid with lattice spacing $d x=\pi / 16$ and time increment $d t=0.01$.

Simulations of Eq. (22) starting from a film with a straight stripe geometry are shown in Fig. 8. We use the exponential potential, with $\bar{h}=1$, and $\theta_{\mathrm{eq}}=60^{\circ}$ (similar results are obtained with a larger thickness $h=1.5$, as reported in Appendix G). The simulations confirm that the pinching process occurs periodically, leaving a periodic array of straight lines. Simulations do not show any important effect of the instability along the front described in Refs. [41,45,46], which was expected by these authors to give rise to fingering, i.e., to the formation of linear structures perpendicular to the dewetting front. Hence the mass shedding process is found to be faster than the fingering instability within our isotropic model and in the range of thicknesses that we have studied. These results justify our one-dimensional description of the periodic mass shedding process reported in the previous sections which discards possible transversal instabilities.

We have also performed similar simulations with additional random roughness in the initial conditions. In order to do so, we have added a random perturbation with uniform distribution in the interval $[-0.025,0.025]$ at each point of the simulation grid. The results are reported in Fig. 9. The initial dynamics is similar to that of simulations without initial roughness. The pinching timescale $t_{\mathrm{ms}}$ and the width of the lines produced by the mass shedding process are only weakly affected by the noise [for example, we have nine lines both in Fig. 8(c) and Fig. 9(c)]. However, at larger times, the stripes break up into dots as expected from the RayleighPlateau instability [3]. The Rayleigh-Plateau instability by which a linear structure breaks up into dots is driven by the 


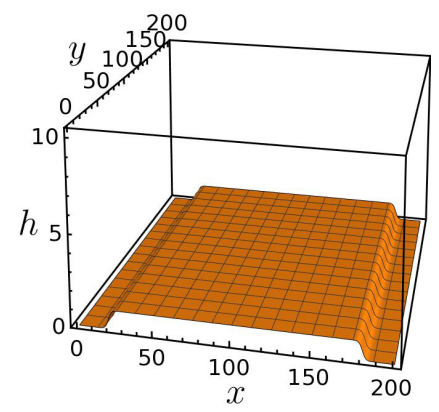

(a)

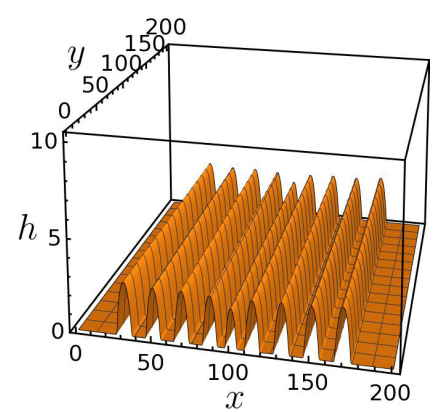

(c)

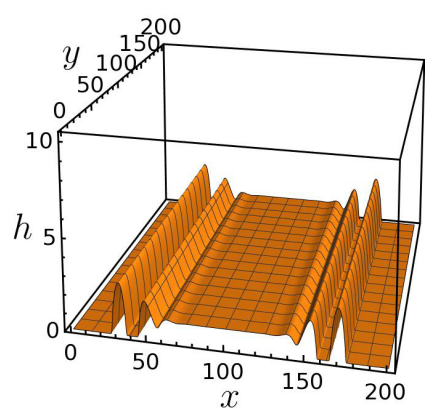

(b)

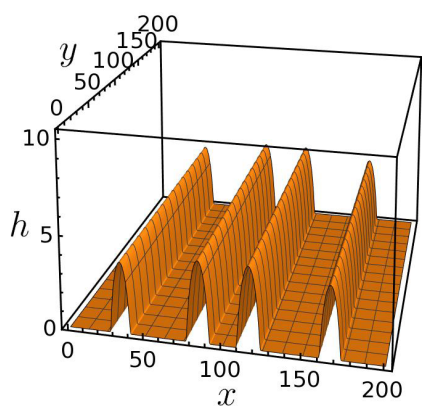

(d)
FIG. 8. Evolution of a stripe. Plots from (a) to (d) correspond to times 0, 200, 600, and 20000, respectively. Simulations are performed using the exponential potential with range $h_{0}=0.1$ and contact angle $\theta_{\mathrm{eq}}=\pi / 3$. The initial thickness of the stripe is $\bar{h}=1$. The edges of the stripe are exponential sigmoids of unit width. Note that the vertical scale has been magnified as compared to the horizontal scale for a better visualization of the surface profile.

continuous reduction of the energy of the line when increasing the amplitude of a modulation of its width (to avoid confusion, we use the term pinching only for the mass-shedding process, and not for the breakup of linear structures). At long times, Ostwald ripening of the islands takes place, where larger islands grow at the expense of smaller ones. Some consequences of the wetting potential on this coarsening process have been investigated in Ref. [28].

The surface roughness in the initial conditions is expected to promote the instabilities such as the fingering and RayleighPlateau instabilities. Indeed, Fourier modes of small transversal perturbations along $y$ with wave vector $q$ are expected to grow as $\zeta_{q}(t)=\zeta_{q}(0) \exp [i \omega t]$, where the dependence of $i \omega$ on $q$ depends on the type of instability. Ignoring nonlinear effects, the time for the amplitude to reach a finite value that could lead to the film breakup $\zeta_{q}(t)=\bar{h}$ is therefore approximated as $t_{\text {inst }}=\ln \left[\bar{h} / \zeta_{q}(0)\right] / i \omega$. The amplitude $\zeta_{q}(0)$ is proportional to roughness in the initial conditions. Thus larger initial roughness leads to smaller $t_{\text {inst }}$. However, the mass shedding process occurs even if the roughness vanishes (it is only triggered by the presence of the edge of the film). Hence to leading order, for small roughness in the initial conditions, the fingering and Rayleigh-Plateau instabilities should appear earlier as compared to mass shedding when increasing amplitude of the roughness. As seen in Figs. 8 and 9 this interpretation is consistent with the observation of

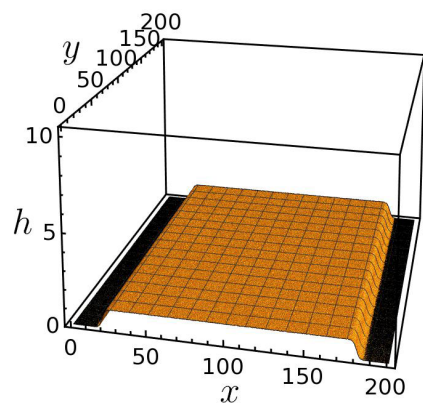

(a)

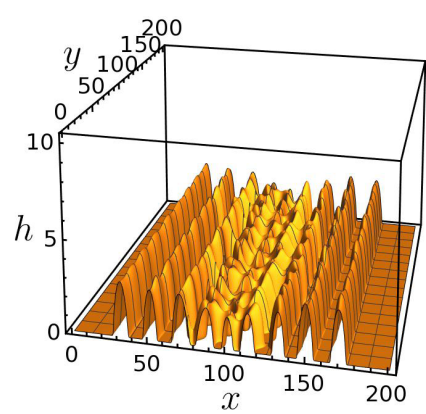

(c)

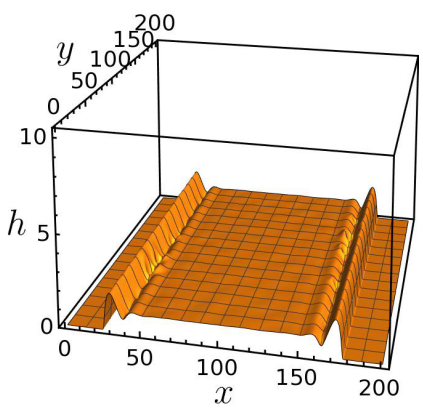

(b)

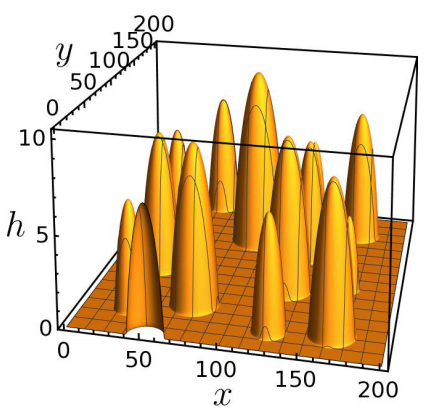

(d)
FIG. 9. Evolution of a rough stripe. Plots from (a) to (d) correspond to times 0,100,400, and 20000, respectively. Same parameters as in Fig. 8, with an additional random roughness in the initial condition.

the strengthening of the Rayleigh-Plateau instability in the simulation results when the initial roughness is increased. However, even in the presence of noise, no fingering is observed irrespective of the noise amplitude. Hence we conclude that within our isotropic model, and within the range of thicknesses that we have studied, the transversal instability during dewetting only gives rise to a Rayleigh-Plateau-like modulation of the rim.

The scenario with formation of lines via periodic pinching and subsequent breakup into islands can also be observed with a circular front geometry. Indeed, as reported in Fig. 10, simulations starting from a small circular hole show a similar behavior with the formation of an array of rings that ultimately break up into dots. Here, the meeting of the mass shedding rings via the periodic boundary conditions initiates the breakup of the rings into dots. However, as reported in Ref. [10], simulations with an isotropic phase field model show that the dewetting of square islands can give rise to more complex patterns.

Simulation with a thinner initial thickness $\bar{h}=0.5$ leads to a destabilization of the film away from the edge due to the spinodal instability, as shown in Fig. 11. Using the expression of time of appearance of the instability given above, we obtain $t_{\text {inst }}^{\mathrm{SD}}=t^{\mathrm{SD}} \ln \left[\bar{h} / \zeta_{q}(0)\right] / 2 \pi$, where $t^{\mathrm{SD}}$ is defined in Eq. (16). Assuming that $\zeta_{q}(0)$ can be approximated by the roughness of the initial perturbation, which is uniformly distributed in the interval $[-\epsilon, \epsilon]$, we obtain $\zeta_{q}(0)=\epsilon / 3^{1 / 2}$. Using this expression, we find that, for the value $\epsilon=0.025$ used in simulations, $t_{\text {inst }}^{\mathrm{SD}} \approx 1.5 \times 10^{2}$ for $\bar{h}=1$ and $t_{\text {inst }}^{\mathrm{SD}}=0.8$ for 


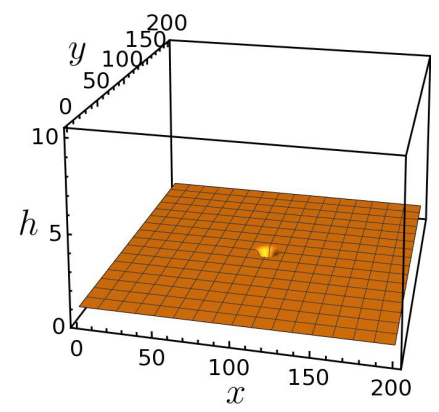

(a)

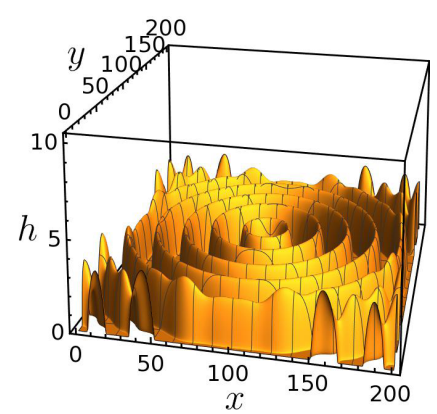

(c)

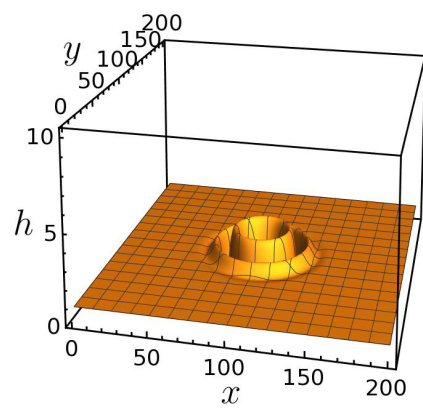

(b)

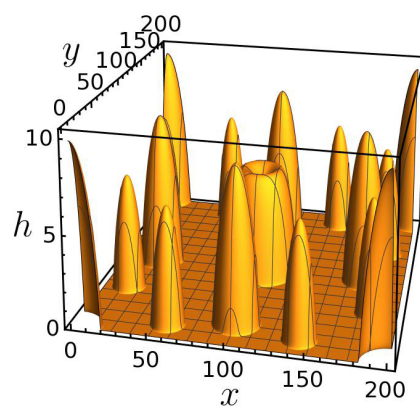

(d)
FIG. 10. Evolution of starting from a circular hole. Same parameters as in Fig. 8. Plots from (a) to (d) correspond to times 0, 200, 1000 , and 20000, respectively.

$\bar{h}=0.5$. Assuming that the mass shedding time is not strongly affected by the roughness, the number of mass shedding events before the spinodal instability comes into play can be evaluated approximately as $n_{\mathrm{ms}}=t_{\text {inst }}^{\mathrm{SD}} / t_{\mathrm{ms}}$. Using the values of $t_{\mathrm{ms}}=118$ for $\bar{h}=1$ and $t_{\mathrm{ms}} \approx 4$ for $\bar{h}=0.5$ obtained from simulations without noise, we find $n_{\mathrm{ms}}=1.28$ for $\bar{h}=1$ and $n_{\mathrm{ms}}=0.2$ for $\bar{h}=0.5$. These results are in qualitative agreement with the simulations, where a few mass shedding events can be observed for $\bar{h}=1$ in Fig. 9, while the spinodal instability takes over before mass shedding occurs for $\bar{h}=0.5$ in Fig. 11.

\section{DISCUSSION AND CONCLUSIONS}

In summary, dewetting dynamics of ultrathin solid films is strongly affected by the wetting potential. In the case of a wetting potential dominated by van der Waals forces, the periodic mass shedding process can be accelerated by orders of magnitude. The mass shedding time $t_{\mathrm{ms}}$ is found to scale with film thickness approximately as $\bar{h}^{6}$. In the case of exponential wetting potentials, we obtain a similar acceleration. However, no simple effective power-law scaling of $t_{\mathrm{ms}}$ with $\bar{h}$ was found in this case. Simulations of the full two-dimensional dynamics confirm that periodic mass shedding occurs, and no fingering instability is observed within our isotropic model. In addition, spinodal dewetting can compete with mass shedding at very small film thicknesses.

Our study neglects the consequences of anisotropy and of the thickness dependence of the mobility. These ingredients should be important for the pinching dynamics, as suggested

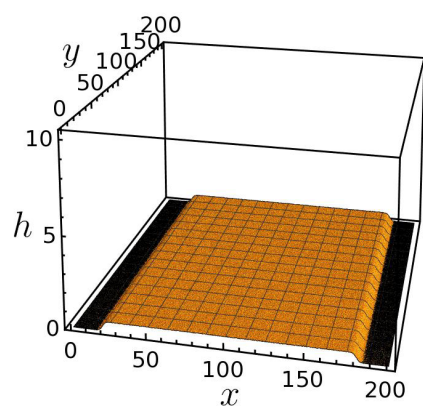

(a)

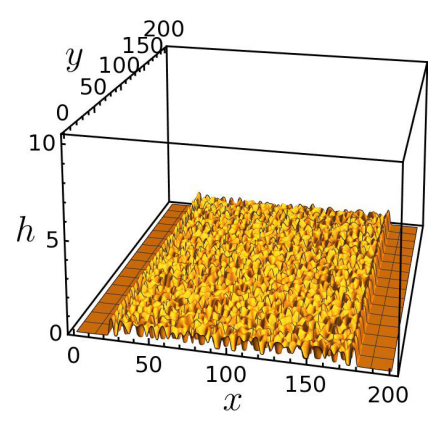

(c)

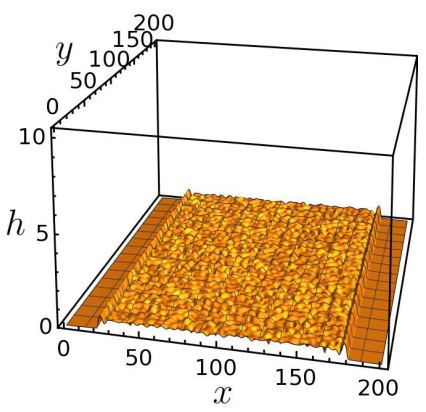

(b)

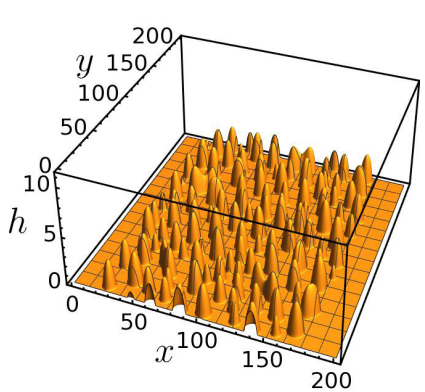

(d)
FIG. 11. Evolution of a very thin film $\bar{h}=0.5$ subject to a strong spinodal instability. Initial conditions with initial roughness and same parameters as in Fig. 9. Plots from (a) to (d) correspond to times 0 , 2,4 , and 200 , respectively.

from previous studies of pinching with anisotropy [27] or thickness dependent mobilities $[47,48]$. Including these effects is an important direction for future theoretical investigations. However, it is also a major challenge for experiments since anisotropy and mobility are most of the time not known accurately for a given experimental system.

Ye and Thompson have reported experimental observations of the motion of solid-state dewetting fronts for $120 \mathrm{~nm}$ thick $\mathrm{Ni}(110)-\mathrm{MgO}$ films. By monitoring the time evolution of a rectangular hole, they observe the thickening of rim near the hole edges and also the pinch-off process $[11,12]$. They also observed the formation of a periodic array of thickened rims due to the pinch-off process. The pinching time observed in their experiments is $t_{\mathrm{ms}} \approx 1255 \mathrm{~min}$ for a film thickness $\bar{h}=120 \mathrm{~nm}$. For Ni-MgO film, the surface energies at $900^{\circ} \mathrm{C}$ are $\gamma_{\mathrm{Ni}} \approx 1.95 \mathrm{~J} / \mathrm{m}^{2}, \gamma_{\mathrm{MgO}} \approx 1.15 \mathrm{~J} / \mathrm{m}^{2}$, and $\gamma_{\mathrm{Ni}-\mathrm{MgO}} \approx 1.12 \mathrm{~J} / \mathrm{m}^{2}$ [49-52]. Thus the equilibrium contact angle is $\theta_{\mathrm{eq}} \approx 90^{\circ}$. Surface mobility can be estimated as $m \approx 5 \times 10^{-29} \mathrm{~m}^{4} / \mathrm{s}$ [53]. Using these physical parameters, the mass shedding time in the absence of wetting potential is found to be $t_{\mathrm{ms}}^{\mathrm{M}} \approx 3604 \mathrm{~min}$, which is three times larger than the experimental result $t_{\mathrm{ms}}^{\mathrm{expt}}$. In order to make the comparison with our numerical results, we assume an exponential potential with range $h_{0}$ of about 2-3 monolayers, which is approximately $1 \mathrm{~nm}$. Using the numerical data obtained for exponential potential with $h_{0}=0.1$, we obtain $t_{\mathrm{ms}} \approx 1097$ for $\bar{h}=120 \mathrm{~nm}$. The experimental result shows a reasonable agreement with our numerical estimation of mass shedding time. However, it should be emphasized that our model is not 
very accurate because the value of $t_{\mathrm{ms}}^{\mathrm{NS}}$ is calculated in the small slope limit [54] and depends on our choice for the value of $h_{0}$. In addition, as discussed above, our analysis neglects the effect of anisotropy and of the possible dependence of the mobility on the film thickness, which is expected to affect the dynamics of dewetting. However, for smaller thicknesses mass shedding should be accelerated by several orders of magnitude. For example, $t_{\mathrm{ms}}^{\mathrm{M}}=109 \mathrm{~min}$ and $t_{\mathrm{ms}}^{\mathrm{NS}}=14 \mathrm{~min}$ for $\bar{h}=50 \mathrm{~nm}$ and $t_{\mathrm{ms}}^{\mathrm{M}}=2.8 \mathrm{~min}$ and $t_{\mathrm{ms}}^{\mathrm{NS}}=0.12 \mathrm{~min}$ for $\bar{h}=20 \mathrm{~nm}$. Hence the effect of the wetting potential should be easier to measure for films that are thinner than $100 \mathrm{~nm}$. We therefore hope that our work will motivate novel directions in the experimental investigations of the dewetting dynamics of ultrathin solid films.

\section{ACKNOWLEDGMENT}

We acknowledge support from the ANR LOTUS Grant (No. ANR-12-BS04-0008).

\section{APPENDIX A: EVOLUTION OF $\boldsymbol{h}_{\text {min }}$ DERIVED FROM THE WORK OF WONG et al. [19]}

We define the dimensionless variable

$$
\zeta=\left(m \Omega \bar{\gamma} \frac{t \alpha_{0}^{4}}{\bar{h}^{4}}\right)^{1 / 5}
$$

where $\alpha_{0}=\theta_{\text {eq. }}$. In the limit of large times and small contact angles, the solution of Ref. [19] to second order provides a quantitative estimate of the film profile. Here, we do not report the derivation of this solution which is provided in detail in Ref. [19]. Using these results, we have obtained the expansion $h_{\text {min }}$ of the film thickness at the first minimum to second order:

$$
\begin{aligned}
\frac{h_{\min }}{\bar{h}}= & 1+\frac{e^{-4 \pi 3^{-3 / 2}}}{72}\left[57+32 \pi 3^{1 / 2}-36 \times 2^{4 / 5} \times 5^{1 / 5} \zeta\right] \\
& +\frac{e^{-4 \pi 3^{-3 / 2}}}{104976 \times 2^{4 / 5} \times 5^{1 / 5} \zeta}\left[131463-35856 \pi 3^{1 / 2}\right. \\
& -120528 \pi^{2}-7680 \pi^{3} 3^{1 / 2}+4096 \pi^{4} \\
& \left.-4\left(81+30 \pi 3^{1 / 2}-32 \pi^{2}\right)^{2}\right] .
\end{aligned}
$$

\section{APPENDIX B: SELF-SIMILAR PINCHING SOLUTION FOR POWER-LAW POTENTIAL}

Neglecting the repulsion terms or other subdominant contributions for large enough $\bar{h}$, we assume a power-law wetting potential

$$
w(h)=-\frac{A}{h^{n}}
$$

Defining the new time variable $\tau=t_{\mathrm{ms}}-t$ the small-slope equation (9) for a power-law potential reads

$$
\begin{aligned}
-\frac{1}{m \Omega} \partial_{\tau} h= & -\bar{\gamma} \partial_{x x x x} h+\left(\partial_{x} h\right)^{2} \frac{A n(n+1)(n+2)}{h^{n+3}} \\
& -\partial_{x x} h \frac{A n(n+1)}{h^{n+2}} .
\end{aligned}
$$

The following ansatz makes all terms scale in the same way with time:

$$
h(x, t)=\left(\frac{A(\Omega m)^{1 / 2}}{\bar{\gamma}^{1 / 2}}\right)^{1 /(n+2)} \tau^{1 /[2(n+2)]} f\left(\frac{x-x_{\mathrm{ms}}}{[m \Omega \gamma \tau]^{1 / 4}}\right) .
$$

This leads to the following expression of the exponents defined in the main text:

$$
\begin{gathered}
\alpha=\frac{1}{4}, \\
\beta=2(n+2),
\end{gathered}
$$

which leads to $\alpha=1 / 4$ and $\beta=8$ in the special case $n=2$ discussed in the main text.

Note that, here, we do not worry about the possibility that some values of $n$ actually may not provide a meaningful solution. In addition, the function $f$ obeys an ordinary differential equation that we do not solve. Instead, we simply observe that this ansatz compares well with the simulations and use $f(0)$ as a fitting parameter in the main text.

\section{APPENDIX C: TOTAL MASS SHEDDING TIME FOR POWER-LAW POTENTIAL: MATCHING PROCEDURE}

We use the two conditions of equal thickness and time derivative of the thickness at $t=t_{*}$ to match the solution in the absence of wetting potential Eq. (A2) and the pinching scaling solution Eq. (B3). In this calculation, we neglect the term $\sim \zeta^{-1}$ in Eq. (A2). This leads to two equations that provide $t_{\mathrm{ms}}$ and $\bar{h}$ as a function of $\zeta_{*}=\left(t_{*} \alpha_{0}^{4} / \bar{h}^{4}\right)^{1 / 5}$ :

$$
\begin{aligned}
\bar{h}= & f(0)^{n /(n+2)}\left(\frac{A(\Omega m)^{1 / 2}}{\bar{\gamma}^{1 / 2}}\right)^{1 / n}\left(\frac{5^{1 / 5} \zeta_{*}}{(n+2)^{1 / 4} \alpha_{0} 2^{1 / 5}}\right)^{2 / n} \\
& \times\left(1+\frac{e^{-4 \pi 3^{-3 / 2}}}{72}\left[57+32 \pi 3^{1 / 2}-36 \times 2^{4 / 5} 5^{1 / 5} \zeta_{*}\right]\right)^{-1-3 /(2 n)}, \\
t_{\mathrm{ms}}= & \frac{1}{m \Omega \bar{\gamma}}\left(\frac{\bar{h} \zeta_{*}}{\alpha_{0}}\right)^{4}\left[\frac{5^{4 / 5}}{(n+2)} \frac{1+\frac{1}{72} e^{-4 \pi 3^{-3 / 2}}\left(57+32 \pi 3^{1 / 2}\right)}{e^{-4 \pi 3^{-3 / 2}} 2^{4 / 5}}\right. \\
& \left.+\zeta_{*}\left(1-\frac{5}{2(n+2)}\right)\right] .
\end{aligned}
$$

The discussions in the main text refer only to the case $n=2$. The mass shedding time $t_{\mathrm{ms}}$ is finally plotted as a function of $\bar{h}$ using a parametric plot with parameter $\zeta_{*}$.

\section{APPENDIX D: PINCHING WITH FINITE RANGE}

Assuming that the potential-dominated regime occurs when $h_{\min }=h_{c}$, and that the pinching in this final regime takes a time $t_{\text {exp }}$, we find

$$
\frac{h_{c}}{\bar{h}}-1=\frac{e^{-4 \pi 3^{-3 / 2}}}{72}\left[57+32 \pi 3^{1 / 2}-36 \times 2^{4 / 5} 5^{1 / 5} \zeta_{\exp }\right] \text {, }
$$




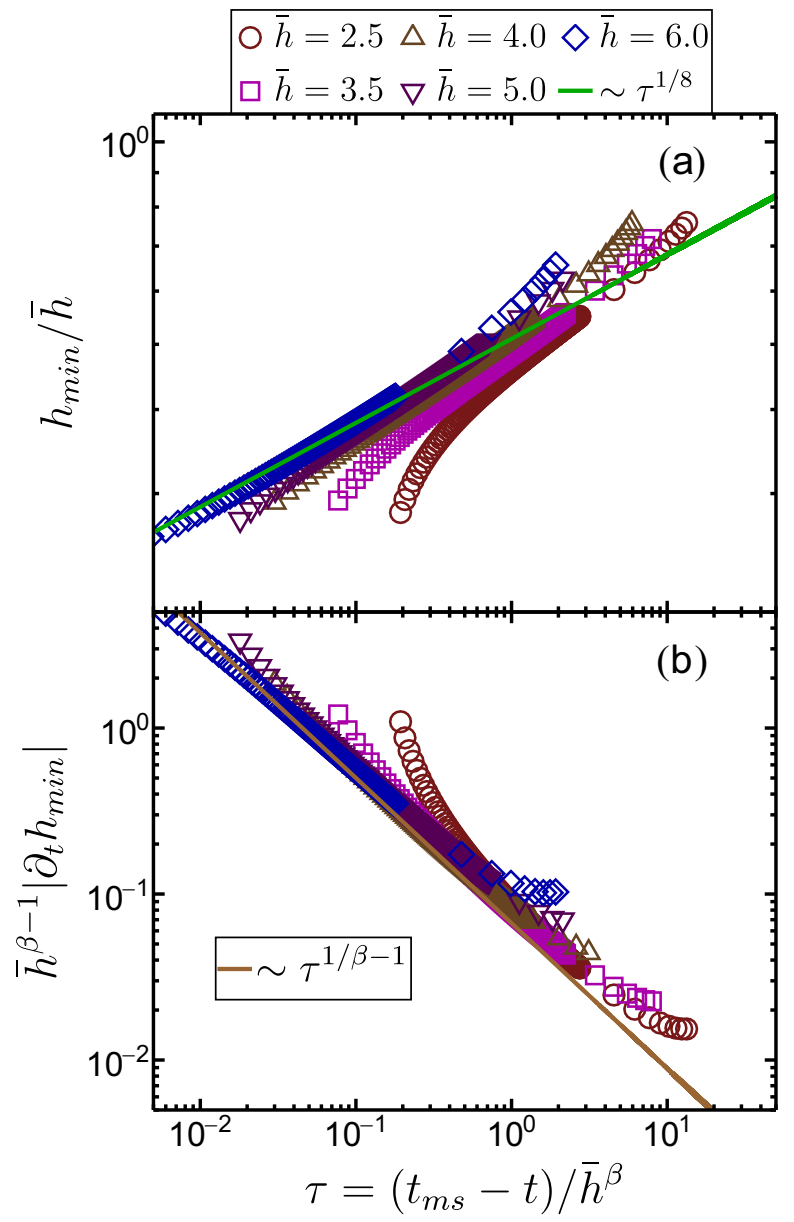

FIG. 12. Data collapse for $h_{\min }$ and its derivative in the pinchoff regime. Data are scaled according to the self-similar solution described in the text with $\beta=8$. The best fit is obtained for $f(0)=1.07$.

with $\zeta_{\exp }=\left[m \Omega \bar{\gamma}\left(t_{\mathrm{ms}}-t_{\mathrm{exp}}\right) \alpha_{0}^{4} / \bar{h}^{4}\right]^{1 / 5}$. This relation is rewritten as

$$
\begin{aligned}
t_{\mathrm{ms}}= & t_{\exp }+\frac{\bar{h}^{4}}{m \Omega \bar{\gamma} \alpha_{0}^{4}} \frac{1}{36^{5} \times 2^{4} 5} \\
& \times\left[57+32 \pi 3^{1 / 2}+\left(1-\frac{h_{c}}{\bar{h}}\right) 72 e^{4 \pi 3^{-3 / 2}}\right]^{5} .
\end{aligned}
$$

This equation is rewritten in the main text in Eq. (21), together with the numerical values of the constants.

\section{APPENDIX E: SELF-SIMILAR SCALING WITH $\beta=8$}

In Fig. 12, we present the rescaled data for $h_{\min }$ and its derivative when imposing the exponent $\beta=8$. A fit of the collapsed data using Eq. (18) provides $f(0)=1.07$ as discussed in the main text.

\section{APPENDIX F: ANALYSIS OF $t_{\mathrm{ms}}$ AND $h_{\text {min }}$ USING $\alpha_{0}=\tan \theta_{\text {eq }}$}

The small slope limit assumes $\alpha_{0} \approx \theta_{\mathrm{eq}} \approx \tan \left(\theta_{\mathrm{eq}}\right)$. When comparing the result of the small slope solution to our

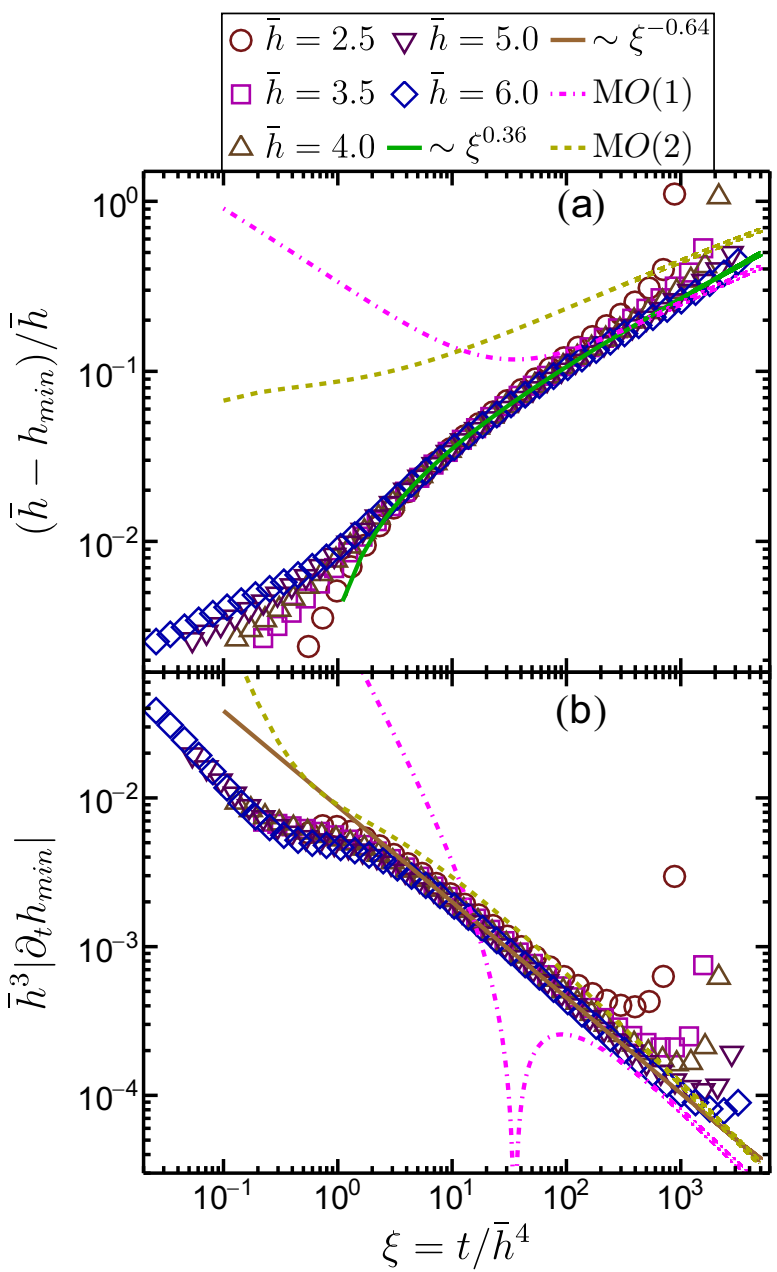

FIG. 13. Simulation data for $h_{\min }$ and its derivative for the van der Waals potential in the Mullins regime. Continuous lines are fit to the data according to the function $\mathcal{F}(\xi)=a+b \xi^{c}$. $\mathrm{MO}(1)$ and $\mathrm{M} O(2)$ represent respectively the first and second order solutions of the small slope approximation of Mullins' model in the long time limit using $\alpha_{0}=\tan \left(\theta_{\mathrm{eq}}\right)$.

numerical data with a contact angle which is not small $\left(\theta_{\mathrm{eq}}=\right.$ $60^{\circ}$ ), we therefore have the choice to choose $\alpha_{0}=\theta_{\mathrm{eq}}$ or $\alpha_{0}=\tan \theta_{\text {eq }}$. The former choice has been used in the main text. For the sake of comparison, we report here some analysis of the numerical simulations for the van der Waals potential using $\alpha_{0}=\tan \theta_{\text {eq }}$.

However, the solution of Wong et al. [19] with $\alpha_{0}=\theta_{\text {eq }}$ (figures in the main text) shows a small deviation from the corresponding solution for $\alpha_{0}=\tan \left(\theta_{\mathrm{eq}}\right)$. To show this, we have included the following results.

First, the numerical results for $h_{\min }$ and its derivative are compared with the prediction of Wong et al. [19] in Fig. 13 assuming $\alpha_{0}=\tan \left(\theta_{\mathrm{eq}}\right)$.

Second, the analysis of the numerical data for the mass shedding time $t_{\mathrm{ms}}$ using $\alpha_{0}=\tan \left(\theta_{\mathrm{eq}}\right)$. The best fit of the matching solution to the numerical data for $t_{\mathrm{ms}}$ provides $f(0)=1.05$, as shown in Fig. 14. 


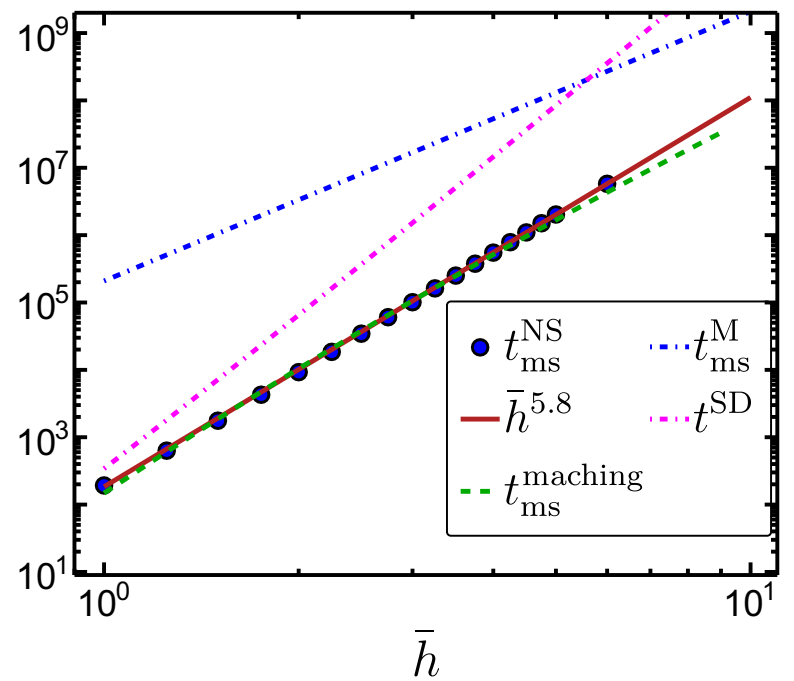

FIG. 14. Mass shedding time $t_{\mathrm{ms}}^{\mathrm{NS}}$ as a function of film thickness for van der Waals potential, analyzed using $\alpha_{0}=\tan \left(\theta_{\mathrm{eq}}\right)$. Here, $t_{\mathrm{ms}}^{\mathrm{M}}$ is the mass shedding time obtained in the absence of wetting potential and $t^{\mathrm{SD}}$ is the timescale of spinodal dewetting. $t_{\mathrm{ms}}^{\text {matching }}$ denotes the matching solution Eqs. (C1) and (C2) using $\alpha_{0}=\tan \left(\theta_{\mathrm{eq}}\right)$. The corresponding fitting parameter is $f(0)=1.05$.

\section{APPENDIX G: TWO-DIMENSIONAL SIMULATIONS WITH $\bar{h}=1.5$}

In Figs. 15 and 16, we report simulations starting from a stripe with initial thickness $\bar{h}=1.5$. All other parameters are

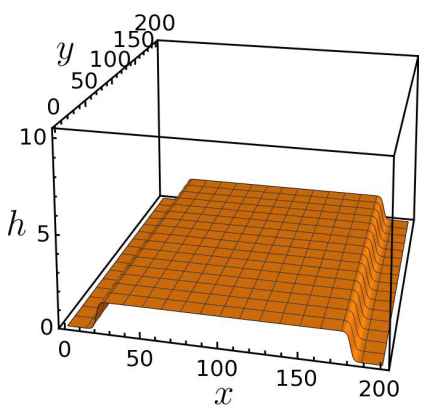

(a)

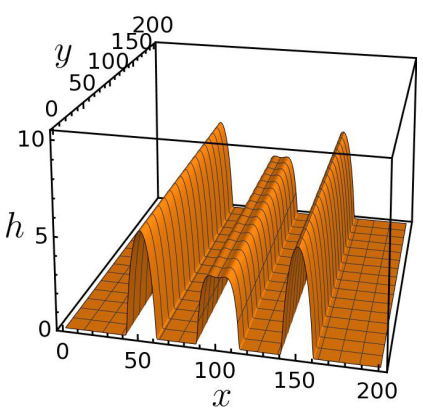

(c)

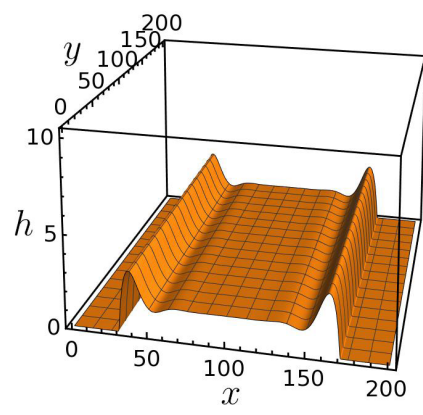

(b)

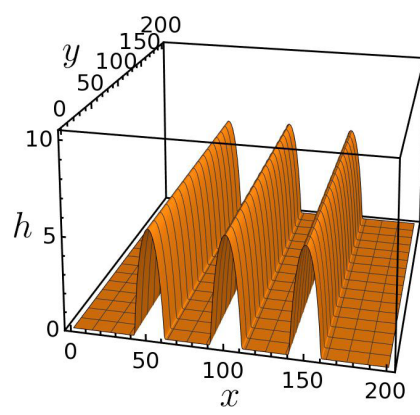

(d)
FIG. 15. Evolution of a stripe with initial thickness $\bar{h}=1.5$. All other parameters are identical to those of Fig. 8. Plots from (a) to (d) correspond to times $0,1000,5000$, and 20000, respectively.

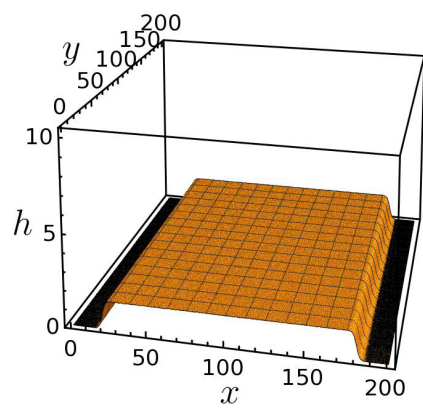

(a)

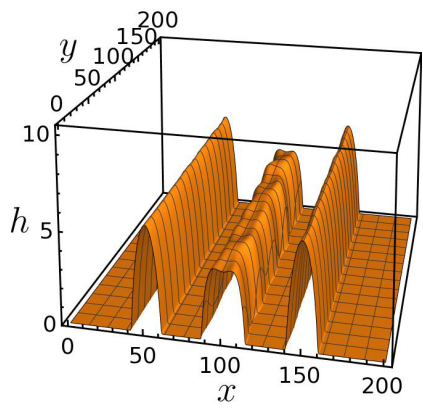

(c)

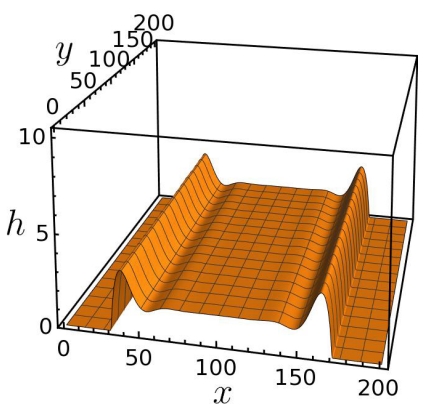

(b)

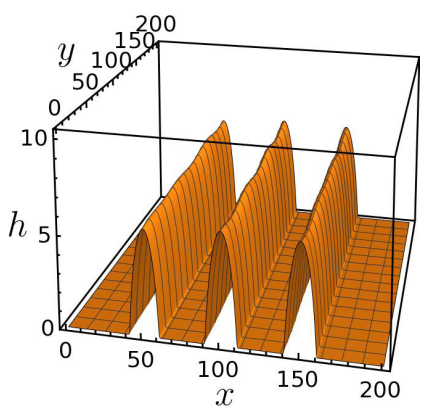

(d)
FIG. 16. Evolution of a rough stripe. All other parameters are identical to those of Fig. 9. Plots from (a) to (d) correspond to times $0,1000,5000$, and 20000 , respectively.

identical to those of Figs. 8 and 9. We observe that the number of lines produced by the mass shedding process is not affected by the initial roughness. Furthermore, the initial roughness leads to the enhancement of the instability along the lines. At longer times, these lines break up into islands.

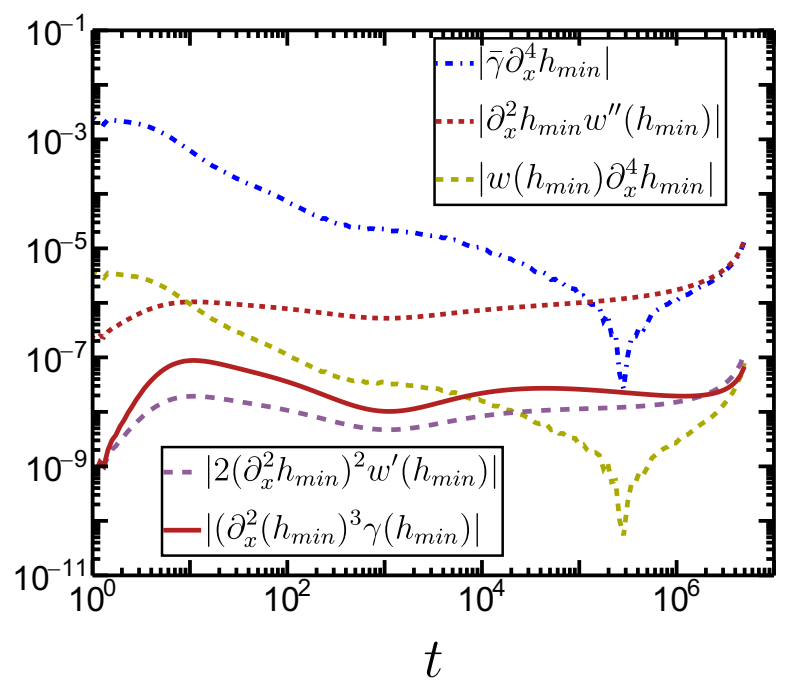

FIG. 17. Comparison of the terms on the right hand side of Eq. (H1) for van der Waals potential. The film thickness is $\bar{h}=6.0$. 


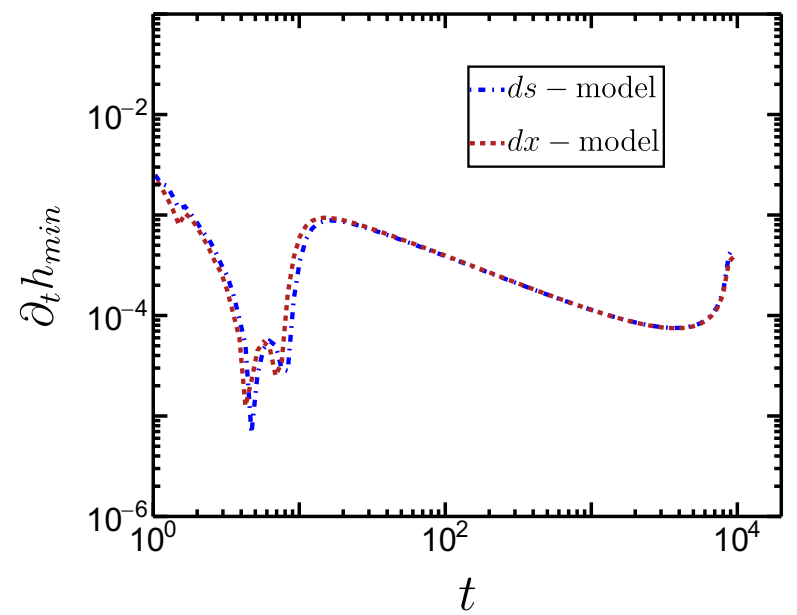

FIG. 18. Comparison of $d s$ model and $d x$ model in 1D. Evolution of the minimum thickness $\partial_{t} h_{\text {min }}$ for an initial film thickness $\bar{h}=2$.

\section{APPENDIX H: TIME EVOLUTION OF FIRST MINIMUM FOR FINITE SLOPE}

The evolution of a minimum $h_{\min }$ of $h(x, t)$ is derived within the finite slope model Eq. (8). Once again, terms proportional to $\partial_{x} h_{\min }$ vanish, and we obtain

$$
\begin{aligned}
\partial_{t} h_{\min }= & m \Omega\left[-\bar{\gamma} \partial_{x}^{4} h_{\min }+w^{\prime \prime}\left(h_{\min }\right) \partial_{x x} h_{\min }-w\left(h_{\min }\right) \partial_{x}^{4} h_{\min }\right. \\
& \left.+\gamma(h)\left(\partial_{x x} h_{\min }\right)^{3}-2 w^{\prime}(h)\left(\partial_{x x} h_{\min }\right)^{2}\right] .
\end{aligned}
$$

The finite slope evolution equation of the minimum Eq. (H1) contains three terms in addition to those already present in the small slope limit Eq. (11). A numerical evaluation of these additional terms, shown in Fig. 17, shows that they are negligible as compared to the terms of the small slope equation.

\section{APPENDIX I: SIMULATIONS WITH THE $d x$ MODEL}

In this Appendix, we provide some results obtained with a free energy where the wetting potential $w(h)$ integrated along $d x$ instead of $d s$, as used in the main text. Within the $d x$ model, the chemical potential reads

$$
\mu=\Omega\left[\bar{\gamma} \kappa+w^{\prime}(h)\right]
$$

All simulations performed with this model lead to results that are qualitatively identical and quantitatively very close to those of the $d s$ model reported in the main text. For example, we show the evolution of the minimum thickness of the film for 1D simulations in Fig. 18. In Fig. 19, we also report $2 \mathrm{D}$ evolution computed from the $d x$ model with parameters and initial conditions which are identical to those of Figs. 8 and 9.

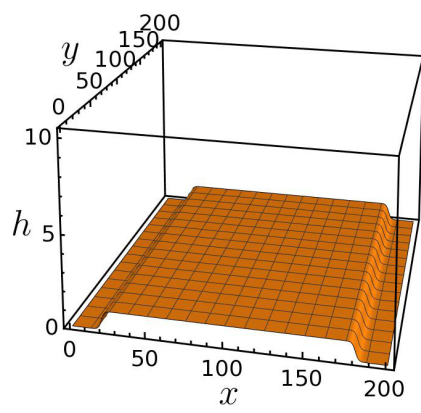

(a)

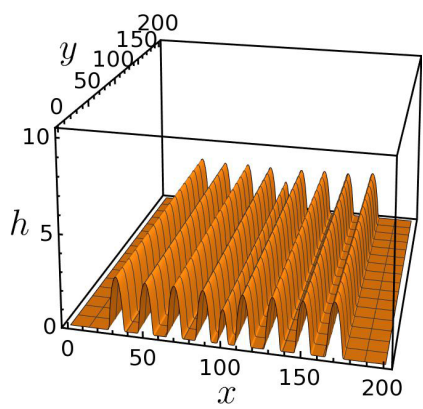

(c)

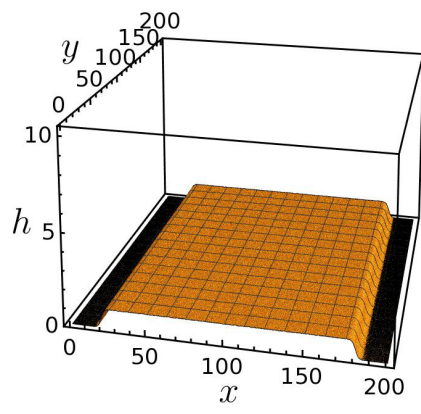

(e)

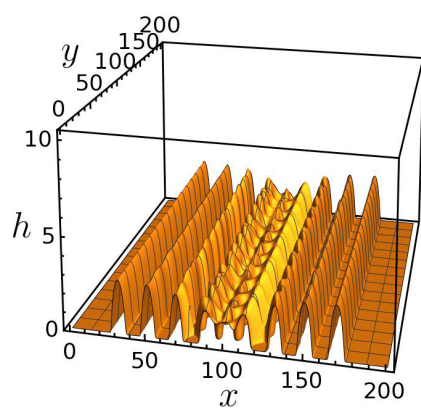

$(\mathrm{g})$

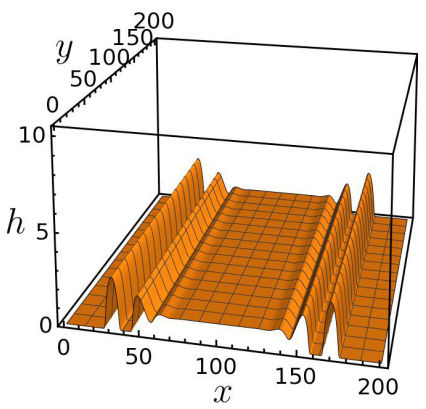

(b)

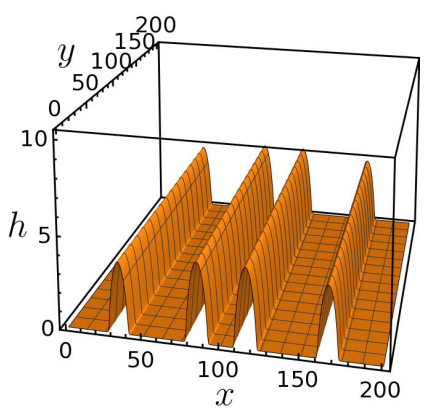

(d)

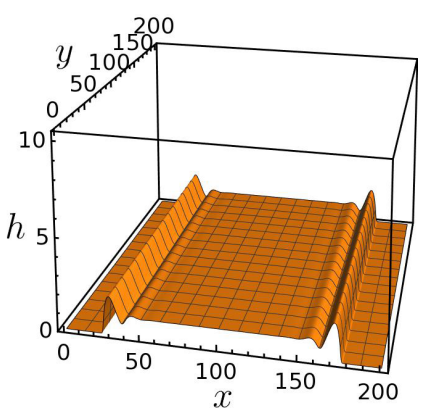

(f)

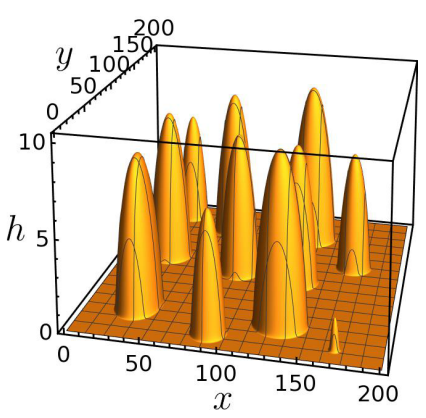

(h)
FIG. 19. Evolution of a stripe with the $d x$ model in 2D. Plots from (a) to (d) refer to the evolution of a stripe without roughness, whereas plots from (e) to (h) describe the evolution of stripe with small initial roughness. All parameters, initial conditions, and evolution time of the plots are identical to those of Fig. 8 without initial roughness and Fig. 9 with initial roughness. 
[1] C. V. Thompson, Annu. Rev. Mater. Res. 42, 399 (2012).

[2] F. Leroy, L. Borowik, F. Cheynis, Y. Almadori, S. Curiotto, M. Trautmann, J. Barbé, and P. Müller, Surf. Sci. Rep. 71, 391 (2016).

[3] D. T. Danielson, D. K. Sparacin, J. Michel, and L. C. Kimerling, J. Appl. Phys. 100, 083507 (2006).

[4] E. Dornel, J.-C. Barbé, F. de Crécy, G. Lacolle, and J. Eymery, Phys. Rev. B 73, 115427 (2006).

[5] E. Bussmann, F. Cheynis, F. Leroy, P. Müller, and O. PierreLouis, New J. Phys. 13, 043017 (2011).

[6] F. Leroy, F. Cheynis, T. Passanante, and P. Müller, Phys. Rev. B 85, 195414 (2012).

[7] F. Cheynis, F. Leroy, T. Passanante, and P. Müller, Appl. Phys. Lett. 102, 161603 (2013).

[8] Z. A. Burhanudin, R. Nuryadi, Y. Ishikawa, and M. Tabe, Thin Solid Films 508, 235 (2006).

[9] G. Capellini, G. Ciasca, M. De Seta, A. Notargiacomo, F. Evangelisti, and M. Nardone, J. Appl. Phys. 105, 093525 (2009).

[10] M. Naffouti, R. Backofen, M. Salvalaglio, T. Bottein, M. Lodari, A. Voigt, T. David, A. Benkouider, I. Fraj, L. Favre, A. Ronda, I. Berbezier, D. Grosso, M. Abbarchi, and M. Bollani, Sci. Adv. 3, eaao1472 (2017).

[11] J. Ye and C. V. Thompson, Appl. Phys. Lett. 97, 071904 (2010).

[12] J. Ye and C. V. Thompson, Phys. Rev. B 82, 193408 (2010).

[13] J. Ye and C. V. Thompson, Acta Mater. 59, 582 (2011).

[14] G. H. Kim, R. V. Zucker, J. Ye, W. C. Carter, and C. V. Thompson, J. Appl. Phys. 113, 043512 (2013).

[15] E. Jiran and C. V. Thompson, J. Electron. Mater. 19, 1153 (1990).

[16] E. Jiran and C. V. Thompson, Thin Solid Films 208, 23 (1992).

[17] C. Kennefick and R. Raj, Acta Metall. 37, 2947 (1989).

[18] A. Kosinova, O. Kovalenko, L. Klinger, and E. Rabkin, Acta Mater. 83, 91 (2015).

[19] H. Wong, P. Voorhees, M. Miksis, and S. Davis, Acta Mater. 48, 1719 (2000).

[20] J. Eggers, Phys. Rev. Lett. 80, 2634 (1998).

[21] W. W. Mullins, J. Appl. Phys. 28, 333 (1957).

[22] D. J. Srolovitz and S. A. Safran, J. Appl. Phys. 60, 247 (1986).

[23] Y. Wang, W. Jiang, W. Bao, and D. J. Srolovitz, Phys. Rev. B 91, 045303 (2015).

[24] W. Jiang, Y. Wang, D. J. Srolovitz, and W. Bao, Phys. Rev. Materials 2, 113401 (2018).

[25] O. Pierre-Louis, Prog. Cryst. Growth Charact. Mater. 62, 177 (2016).

[26] A. K. Tripathi and O. Pierre-Louis, Phys. Rev. E 97, 022801 (2018).

[27] M. Khenner, Phys. Rev. B 77, 165414 (2008).
[28] A. Constantinescu, L. Golubović, and A. Levandovsky, Phys. Rev. E 88, 032113 (2013).

[29] J. A. Diez and L. Kondic, Phys. Fluids 19, 072107 (2007).

[30] W. Tewes, M. Wilczek, S. V. Gurevich, and U. Thiele, Phys. Rev. Fluids 4, 123903 (2019).

[31] J. Israelachvili, Intermolecular and Surface Forces (Elsevier Science, Amsterdam, 2015).

[32] C.-h. Chiu, Appl. Phys. Lett. 75, 3473 (1999).

[33] M. Ortiz, E. Repetto, and H. Si, J. Mech. Phys. Solids 47, 697 (1999).

[34] W. T. Tekalign and B. J. Spencer, J. Appl. Phys. 96, 5505 (2004).

[35] J.-N. Aqua, T. Frisch, and A. Verga, Phys. Rev. B 76, 165319 (2007).

[36] N. Tretyakov, M. Müller, D. Todorova, and U. Thiele, J. Chem. Phys. 138, 064905 (2013).

[37] U. Thiele, D. V. Todorova, and H. Lopez, Phys. Rev. Lett. 111, 117801 (2013)

[38] J.-N. Aqua and T. Frisch, Phys. Rev. B 82, 085322 (2010).

[39] B. Dai, L. G. Leal, and A. Redondo, Phys. Rev. E 78, 061602 (2008).

[40] G. McFadden, S. Coriell, and R. Sekerka, J. Cryst. Growth 91, 180 (1988).

[41] M. Dziwnik, M. Korzec, A. Münch, and B. Wagner, Multiscale Model. Simul. 12, 755 (2014).

[42] W. W. Zhang and J. R. Lister, Phys. Fluids 11, 2454 (1999).

[43] T. P. Witelski and A. J. Bernoff, Phys. Fluids 11, 2443 (1999).

[44] D. Vaynblat, J. R. Lister, and T. P. Witelski, Phys. Fluids 13, 1130 (2001).

[45] W. Kan and H. Wong, J. Appl. Phys. 97, 043515 (2005).

[46] M. Dufay and O. Pierre-Louis, Phys. Rev. Lett. 106, 105506 (2011).

[47] J. R. King and M. Bowen, Eur. J. Appl. Math. 12, 321 (2001).

[48] A. Münch, B. A. Wagner, and T. P. Witelski, J. Eng. Math. 53, 359 (2005).

[49] T. Barsotti, J. Bermond, and M. Drechsler, Surf. Sci. 146, 467 (1984).

[50] P. Lazar and M. Otyepka, Phys. Rev. B 91, 115402 (2015).

[51] D. Matsunaka and Y. Shibutani, Phys. Rev. B 77, 165435 (2008).

[52] J. Chen and N. Chen, J. Phys.: Condens. Matter 22, 215001 (2010).

[53] J. Blakely and H. Mykura, Acta Metall. 9, 23 (1961).

[54] The value for $t_{\mathrm{ms}}^{\mathrm{NS}}$ is obtained from finite slope simulations with $\theta_{\mathrm{eq}}=60^{\circ}$. Then, the mass shedding time for Ni film with $\theta_{\mathrm{eq}}=$ $90^{\circ}$ is evaluated from the assumption $t_{\mathrm{ms}}^{\mathrm{NS}} \propto \theta_{\mathrm{eq}}^{4}$. 